\title{
New Guanidinium-based Room-temperature Ionic Liquids. Substituent and Anion Effect on Density and Solubility in Water
}

\author{
Milen G. Bogdanov ${ }^{\mathrm{a}, \mathrm{c}}$, Desislava Petkova ${ }^{\mathrm{a}, \mathrm{c}}$, Stanimira Hristeva ${ }^{\mathrm{a}, \mathrm{c}}$, Ivan Svinyarov ${ }^{\mathrm{a}, \mathrm{c}}$, \\ and Willi Kantlehner ${ }^{b, c}$ \\ ${ }^{a}$ Faculty of Chemistry, University of Sofia, 1, J. Bourchier Blvd., 1164 Sofia, Bulgaria \\ ${ }^{\mathrm{b}}$ Institut für Organische Chemie, Universität Stuttgart, Pfaffenwaldring 55, 70569 Stuttgart, \\ Germany \\ ${ }^{c}$ Fakultät Chemie/Organische Chemie, Hochschule Aalen, Beethovenstraße 1, 73430 Aalen, \\ Germany
}

Reprint requests to Dr. Milen G. Bogdanov. E-mail: mbogdanov@chem.uni-sofia.bg

Z. Naturforsch. 2010, 65b, 37 -48; received October 28, 2009

Dedicated to Professor Klaus Peseke on the occasion of his $70^{\text {th }}$ birthday

\begin{abstract}
In order to examine the influence of the alkyl chain length on some physical properties of guanidinium salts, the synthesis of a homologous series of new $N^{\prime \prime}-n$-alkylsubstituted $N, N$-diethyl- $N^{\prime}, N^{\prime}$ di- $n$-propyl- $N^{\prime \prime}-n$-hexyl guanidinium ionic liquids (gILs), containing chloride $(\mathrm{Cl}$ ), tetrafluoroborate $\left(\mathrm{BF}_{4}\right)$, acesulfamate (Ace), saccharinate $(\mathrm{Sac})$, and tosylate (Tos) as anions, is reported. $\mathbf{C}_{\boldsymbol{n}}$-gILAce, $\mathbf{C}_{\boldsymbol{n}}$-gILSac, and $\mathbf{C}_{\boldsymbol{n}}$ - $\mathbf{g I L B F} \mathbf{H}_{4}$ were obtained by ion exchange reaction of the corresponding hexasubstituted guanidinium chlorides $\left(\mathbf{C}_{\boldsymbol{n}}-\mathbf{g C l}, n=3,4,6,8,10\right)$, which were synthesized by a quaternization reaction of the pentaalkyl-substituted guanidine $\mathbf{3}$ and the corresponding alkylchloride in DMF. The tosylates gILs $\mathbf{C}_{\boldsymbol{n}}$-gTos $(n=1,2,4,6,8,10)$ were synthesized by alkylation of $\mathbf{3}$ with the corresponding alkyltosylates. Some physical properties, such as solubility in water and organic solvents, refractive index and density, are considered as a function of the length of the $n$-alkyl substituent $\mathrm{R}$ and the nature of the anion.
\end{abstract}

Key words: Ionic Liquids, Guanidinium Salts, Density, Quaternization Reaction, DMF

\section{Introduction}

Room-temperature ionic liquids (RTILs) have attracted enormously the attention of the scientific community in the last two decades. Consisting entirely of ions (normally a small organic cation and an inorganic or organic anion), they are liquids at ambient temperature and display high thermal and chemical stability, a large electrochemical window, high ionic conductivity, relatively low viscosity and negligible vapor pressure. Furthermore, depending on the ion combination, they can be soluble or insoluble in water or organic solvents, respectively. These unique physico-chemical properties favor their application in diverse fields such as synthesis [1a], catalysis [1b], electrochemistry [1c], nanotechnology [1d], separation technology, and analytical chemistry [1e], etc.

Along with ILs based on 1,3-dialkylimidazolium cations, which seem to dominate the ILs area, ILs based on tetraalkylammonium, sulfonium, phosphonium and pyrrolidinium cations have been exten- sively studied. In contrast, the knowledge on the synthesis, physical properties and potential application of ILs based on the guanidinium ion is quite rare. A retrospective view on guanidinium ILs (gILs) in the last decade shows the availability of only a few published reports: two examples of iodide and tricyanomethanide $(\mathrm{NC})_{3} \mathrm{C}^{-}$gILs were used for dyesensitized solar cells [2]; series of gILs, including cyclic representatives, based on $\mathrm{NO}_{3}{ }^{-}, \mathrm{ClO}_{4}{ }^{-}$and $\left(\mathrm{NO}_{2}\right)_{2} \mathrm{~N}^{-}$anions were synthesized as high-energy ILs by Schreeve and coworkers [3]; several examples of dimethyltetraalkyl gILs-based on $\mathrm{BF}_{4}{ }^{-}, \mathrm{PF}_{6}{ }^{-}, \mathrm{NTf}_{2}{ }^{-}$, $\mathrm{Sac}^{-}$, and $\mathrm{N}(\mathrm{CN})_{2}{ }^{-}$anions have been reported as a new generation of RTILs by Afonso and coworkers $[4 a, b]$; more than thirty hexaalkylguanidinium trifluoromethanesulfonates $\left(\mathrm{TfO}^{-}\right)$have been reported by Kunkel and Maas [5], and new chiral hexasubstituted RTgILs by Afonso and coworkers [4c] and Shah and Liebscher [6]; very recently, a series of (bis(trifluoromethylsulfonyl)imide) $\left(\mathrm{Tf}_{2} \mathrm{~N}^{-}\right)$gILs have been reported by Yang and coworkers $[7 \mathrm{a}, \mathrm{b}]$ as poten- 
Table 1. Structure and abbreviation of the synthesized RTILs based on $N^{\prime \prime}$ - $n$-alkyl-substituted $N, N$-diethyl- $N^{\prime}, N^{\prime}$-di- $n$-propyl$N^{\prime \prime}$ - $n$-hexyl guanidinium cations $\left(\mathbf{C}_{\boldsymbol{n}}-\mathbf{g}^{+}\right)$.

\begin{tabular}{|c|c|c|c|c|c|}
\hline$\overline{\mathrm{C}_{n}-\mathrm{g}^{+}}$ & $\begin{array}{l}\text { Cloride } \\
\mathrm{Cl}^{-}\end{array}$ & $\begin{array}{l}\text { Tetrafluoroborate } \\
\mathrm{BF}_{4}^{-}\end{array}$ & $\begin{array}{l}\text { Saccharinate } \\
\mathrm{Sac}^{-}\end{array}$ & $\begin{array}{l}\text { Acesulfamate } \\
\text { Ace }^{-}\end{array}$ & $\begin{array}{l}\text { Tosylate } \\
\text { Tos }^{-}\end{array}$ \\
\hline $\mathrm{C}_{6} \mathrm{H}_{13} \mathrm{C}_{2} \mathrm{H}_{5}$ & $\mathrm{Cl}^{\ominus}$ & $\begin{array}{c}\mathrm{F} \\
\mathrm{F}-\underset{\mathrm{l}}{\mathrm{B}}-\mathrm{F} \\
\mathrm{F} \\
\mathrm{F}\end{array}$ & & $\mathrm{O}^{-} \stackrel{\mathrm{N}}{\mathrm{S}}=$ & \\
\hline $\mathrm{R}=\mathrm{CH}_{3-}^{-}$ & - & - & - & - & $\mathrm{C}_{1}$-gTos \\
\hline $\mathrm{R}=\mathrm{C}_{2} \mathrm{H}_{5}^{-}$ & - & - & - & - & $\mathrm{C}_{2}$-gTos \\
\hline $\mathrm{R}=\mathrm{C}_{3} \mathrm{H}_{7}-$ & $\mathrm{C}_{3}-\mathrm{gCl}$ & $\mathrm{C}_{3}-\mathrm{gBF}_{4}$ & $\mathrm{C}_{3}-\mathrm{gSac}$ & $\mathrm{C}_{3}$-gAce & - \\
\hline $\mathrm{R}=\mathrm{C}_{4} \mathrm{H}_{9-}$ & $\mathrm{C}_{4}-\mathrm{gCl}$ & $\mathrm{C}_{4}-\mathrm{gBF}_{4}$ & $\mathrm{C}_{4}-\mathrm{gSac}$ & $\mathrm{C}_{4}$-gAce & $\mathrm{C}_{4}$-gTos \\
\hline $\mathrm{R}=\mathrm{C}_{5} \mathrm{H}_{13}-$ & $\mathrm{C}_{6}-\mathrm{gCl}$ & $\mathrm{C}_{6}-\mathrm{gBF}_{4}$ & $\mathrm{C}_{6}-\mathrm{gSac}$ & $\mathrm{C}_{6}$-gAce & $\mathrm{C}_{6}-\mathrm{gTos}$ \\
\hline $\mathrm{R}=\mathrm{C}_{8} \mathrm{H}_{17}-$ & $\mathrm{C}_{8}-\mathrm{gCl}$ & $\mathrm{C}_{8}-\mathrm{gBF}_{4}$ & $\mathrm{C}_{8}-\mathrm{gSac}$ & $\mathrm{C}_{8}$-gAce & $\mathrm{C}_{8}$-gTos \\
\hline $\mathrm{R}=\mathrm{C}_{10} \mathrm{H}_{21}{ }^{-}$ & $\mathrm{C}_{10}-\mathrm{gCl}$ & $\mathrm{C}_{10}-\mathrm{gBF}_{4}$ & $\mathrm{C}_{10}-\mathrm{gSac}$ & $\mathrm{C}_{10}$-gAce & $\mathrm{C}_{10}$-gTos \\
\hline
\end{tabular}

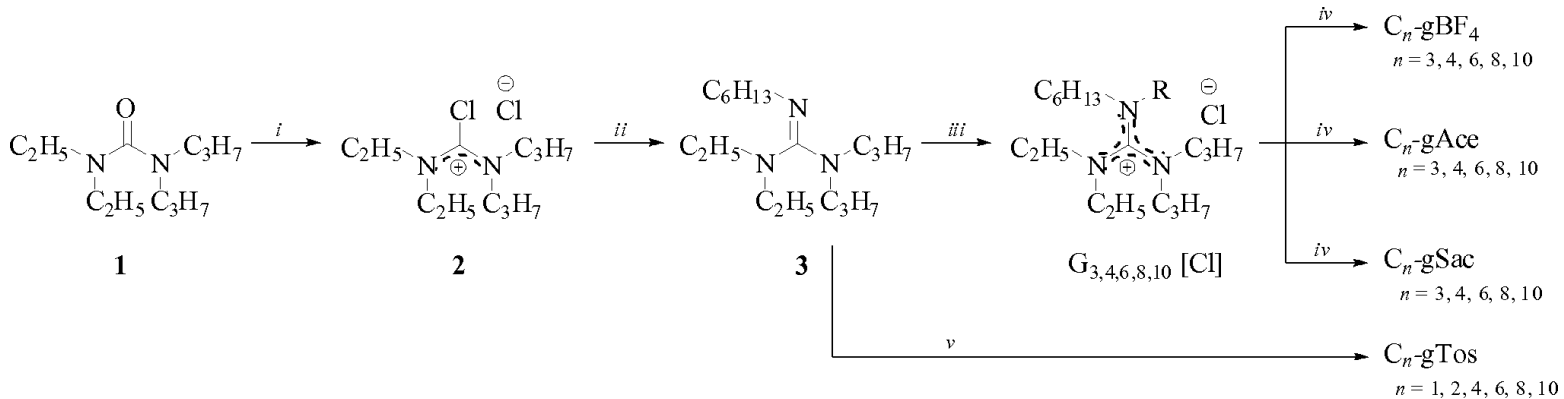

Scheme 1. Synthesis of guanidinium RTILs. Reagents and conditions: (i) $\mathrm{COCl}_{2} / \mathrm{CH}_{3} \mathrm{CN},-25{ }^{\circ} \mathrm{C}$ to r. t., $24 \mathrm{~h}$ (yield $98 \%$ ); (ii) 2 in $\mathrm{CH}_{3} \mathrm{CN} / \mathrm{H}_{2} \mathrm{NC}_{6} \mathrm{H}_{13},-10{ }^{\circ} \mathrm{C}$ to r.t., $16 \mathrm{~h}$ (yield $80 \%$ ); (iii) $\mathrm{RCl} / \mathrm{DMF}, 100-110{ }^{\circ} \mathrm{C}, 10-13 \mathrm{~h}$ (yield $30-87 \%$ ); (iv) $\mathrm{MeOH}$ or water/K or Na salt of the corresponding anion, $60{ }^{\circ} \mathrm{C}, 4 \mathrm{~h}$ (yield $82-99 \%$ ); (v) TosR/DMF, $100-110{ }^{\circ} \mathrm{C}$, $2-4$ h (yield $83-99 \%)$.

tial new electrolytes for lithium batteries, and a new cyclic guanidinium ionic liquid OGI (1,3-dimethyl-2$N^{\prime \prime}$-methyl- $N^{\prime \prime}$-octylimidazoguanidinium iodide) was used as a quasi-solid-state electrolyte for dyesensitized solar cells by Li et al. [8]. It should further be mentioned that as a result of finding a new protocol for the synthesis of hexasubstituted guanidinium salts [9a-d], Kantlehner et al. in 1984 were able to synthesize a large number (more than hundred) of hexaalkylguanidinium salts [9e], which in two cases have been described as oils. Nevertheless, considering the great versatility of the guanidinium cation (we have calculated more than $10^{5}$ possible combinations if one varies the alkyl substituents from methyl to dodecyl in the guanidinium ion) the above contributions represent a "drop in the ocean" in the field of guanidinium-based ILs, or in the ILs area in general. Having in mind this incredible number, a lot of work impends upon the development of new synthetic methods for gILs and the investigation of their properties and potential industrial applications.
In this direction, in order to investigate the influence of the anions and the chain length of the alkyl substituents on some physical properties, here we report the synthesis of a series of hydrophobic room-temperature hexaalkylguanidinium ionic liquids $\left(\mathbf{C}_{\boldsymbol{n}}-\mathbf{g X}\right)$, containing chloride $(\mathrm{Cl})$, tetrafluoroborate $\left(\mathrm{BF}_{4}\right)$, acesulfamate (Ace), saccharinate (Sac), and tosylate (Tos) as anions $\mathrm{X}$ (Table 1).

\section{Results and Discussion}

\section{Synthesis of compounds}

The synthesis of the guanidinium salts is presented in Scheme 1. $N, N$-diethyl- $N^{\prime}, N^{\prime}$-di-n-propylurea $\mathbf{1}$, the corresponding chloroformamidinium chloride $\mathbf{2}$ and guanidine $\mathbf{3}$ were synthesized as previously described by Kantlehner and coworkers [2,9a-e]. In order to synthesize guanidinium halides for anion metathesis reactions, guanidine $\mathbf{3}$ was further quaternized with chloroalkanes instead of bromo- or iodo-substituted analogs. It is known [10] that the bromoalkanes re- 
Table 2. Abbreviations, molecular weights, densities, water content, water uptake ability, and solubility in water and some organic solvents of the synthesized RTgILs evaluated in this study.

\begin{tabular}{|c|c|c|c|c|c|c|}
\hline \multirow[t]{2}{*}{ Compound } & \multirow{2}{*}{$\begin{array}{c}M_{\mathrm{W}} \\
\left(\mathrm{g} \mathrm{mol}^{-1}\right)\end{array}$} & \multirow{2}{*}{$\begin{array}{c}\rho_{\text {dried }}{ }^{\mathrm{a}} \\
\left(\mathrm{g} \mathrm{mL}^{-1}\right)\end{array}$} & \multirow{2}{*}{$\begin{array}{c}\text { Water content }^{\mathrm{b}} \\
(\mathrm{ppm})\end{array}$} & \multirow{2}{*}{$\begin{array}{c}\text { Water uptake } \\
(\text { wt- } \%)\end{array}$} & \multicolumn{2}{|c|}{ Solubility $^{\mathrm{d}}$} \\
\hline & & & & & immiscible & miscible \\
\hline$\overline{\mathrm{C}_{3}-\mathrm{gCl}}$ & 362.04 & - & 670 & soluble & de, ch, bme & $\mathrm{w}$, et, dcm, ac \\
\hline $\mathrm{C}_{4}-\mathrm{gCl}$ & 376.06 & 0.949 & 690 & soluble & de, ch, bme & $\mathrm{w}$, et, dcm, ac \\
\hline $\mathrm{C}_{6}-\mathrm{gCl}^{\mathrm{e}}$ & 404.12 & - & - & soluble & de, ch, bme & w, et, dcm, ac \\
\hline $\mathrm{C}_{8}-\mathrm{gCl}$ & 432.17 & 0.931 & 720 & soluble & de, ch, bme & w, et, dcm, ac \\
\hline $\mathrm{C}_{10}-\mathrm{gCl}$ & 460.22 & 0.929 & 600 & soluble & w, ch & et, dcm, ac de, bme \\
\hline $\mathrm{C}_{3}-\mathrm{gBF}_{4}$ & 413.39 & 1.011 & 370 & 5.21 & w, de, ch, bme & et, $\mathrm{dcm}, \mathrm{ac}$ \\
\hline $\mathrm{C}_{4}-\mathrm{gBF}_{4}$ & 427.41 & 1.009 & 330 & 4.36 & w, de, ch, bme & et, $\mathrm{dcm}, \mathrm{ac}$ \\
\hline $\mathrm{C}_{6}-\mathrm{gBF}_{4}$ & 455.77 & 0.997 & 350 & 3.19 & w, de, ch, bme & et, $\mathrm{dcm}, \mathrm{ac}$ \\
\hline $\mathrm{C}_{8}-\mathrm{gBF}_{4}$ & 483.52 & 0.990 & 330 & 3.03 & w, de, ch, bme & et, dcm, ac \\
\hline $\mathrm{C}_{10}-\mathrm{gBF}_{4}$ & 511.57 & 0.981 & 300 & 2.88 & w, de, ch, bme & et, dcm, ac \\
\hline $\mathrm{C}_{3}$-gAce & 488.73 & 1.056 & 390 & 20.64 & w, de, ch, bme & et, $\mathrm{dcm}, \mathrm{ac}$ \\
\hline $\mathrm{C}_{4}$-gAce & 502.75 & 1.050 & 406 & 17.77 & w, de, ch, bme & et, dcm, ac \\
\hline $\mathrm{C}_{6}$-gAce & 530.81 & 1.031 & 420 & 12.55 & w, de, ch, bme & et, dcm, ac \\
\hline $\mathrm{C}_{8}$-gAce & 558.86 & 1.022 & 350 & 10.88 & w, de, ch, bme & et, $\mathrm{dcm}, \mathrm{ac}$ \\
\hline $\mathrm{C}_{10}$-gAce & 586.45 & 1.005 & 360 & 10.35 & w, de, ch, bme & et, $\mathrm{dcm}, \mathrm{ac}$ \\
\hline $\mathrm{C}_{3}-\mathrm{gSac}$ & 508.76 & 1.069 & 520 & 24.77 & w, de, ch, bme & et, $\mathrm{dcm}, \mathrm{ac}$ \\
\hline $\mathrm{C}_{4}-\mathrm{gSac}$ & 522.79 & 1.063 & 400 & 15.31 & w, de, ch, bme & et, dcm, ac \\
\hline $\mathrm{C}_{6}-\mathrm{gSac}$ & 550.84 & 1.045 & 420 & 12.73 & w, de, ch, bme & et, dcm, ac \\
\hline $\mathrm{C}_{8}-\mathrm{gSac}$ & 578.89 & 1.041 & 350 & 9.15 & w, de, ch, bme & et, dcm, ac \\
\hline $\mathrm{C}_{10}-\mathrm{gSac}$ & 606.95 & 1.024 & 360 & 10.46 & w, de, ch, bme & et, dcm, ac \\
\hline $\mathrm{C}_{1}$-gTos & 469.72 & 1.045 & 650 & soluble & de, ch, bme & w,et, dcm, ac \\
\hline $\mathrm{C}_{2}$-gTos & 483.75 & 1.035 & 740 & soluble & de, ch, bme & $\mathrm{w}$, et, dcm, ac \\
\hline $\mathrm{C}_{4}$-gTos & 511.38 & 1.027 & 750 & soluble & de, ch, bme & $\mathrm{w}$, et, dcm, ac \\
\hline $\mathrm{C}_{6}$-gTos & 539.86 & 1.014 & 350 & 26.78 & w, de, ch, bme & et, dcm, ac \\
\hline $\mathrm{C}_{8}-\mathrm{gTos}$ & 567.91 & 1.001 & 300 & 23.69 & w, de, ch, bme & et, dcm, ac \\
\hline $\mathrm{C}_{10}$-gTos & 595.96 & 0.989 & 450 & 15.93 & w, de & et, dcm, ac, ch, bme \\
\hline
\end{tabular}

${ }^{\text {a }}$ Measured at $25{ }^{\circ} \mathrm{C} ;{ }^{b}$ water content estimated prior to the measurements; ${ }^{\mathrm{c}}$ measured at $25{ }^{\circ} \mathrm{C}$; ${ }^{\mathrm{d}}$ observed two phases (immiscible) and complete solubilization (miscible), where: de - diethyl ether, ch - cyclohexane, bme - butyl methyl ether, w - water, et - ethanol, dcm dichloromethane, ac - acetone; ${ }^{\mathrm{e}}$ not liquid at r. t.

act strongly exothermic as the reaction rate increases, which is a disadvantage for large-scale experiments. The reaction with iodoalkanes can be often carried out at room temperature, but the formed iodide salts are light-sensitive. Moreover, the solubility of many inorganic salts $\mathrm{M}^{+} \mathrm{X}^{-}$in water decreases in the order $\mathrm{Cl}^{-}<\mathrm{Br}^{-}<\mathrm{I}^{-}$, which has a high impact on the anion exchange reaction. It seems that the only disadvantage of using chloroalkanes in this important step is the prolonged reaction time of $2-3 \mathrm{~d}$ [11]. To overcome this problem, appropriate reaction conditions that can accelerate the reaction and increase the yields must be found.

Although different solvents might be used in the quaternization step, the reaction for the ILs purpose is normally carried out under solvent-free conditions, i. e. the two reagents are mixed and heated to give two phases. Furthermore, from the recently reported [12] comparative study on the rate of single-phase and twophase synthesis of BumimCl it can be concluded that the rate of a stirred solvent-free reaction is almost the same as that in a single-phase system containing 20 vol- $\%$ ethanol, which shows that there is no particular advantage in using solvents. On the other hand, it is well known that the nucleophilic substitution reactions $\left(\mathrm{S}_{\mathrm{N}}\right)$ are accelerated in more polar solvents in the case of uncharged species reacting to charged ones [13]. On this basis, it could be assumed that the use of relatively highly polar DMF (dielectric constant $=38.3$, b. p. $153^{\circ} \mathrm{C}$ ) as solvent in the quaternizaton step will accelerate the reaction. To prove the validity of this assumption, independent reactions between the pentasubstituted guanidine $\mathbf{3}$ and $\mathrm{BuCl}$ as reference compounds were carried out on the 0.013 molar scale. Each reaction mixture was heated at $110{ }^{\circ} \mathrm{C}$ (oil bath) for the appropriate reaction time, worked up, and the yields were estimated. Using this strategy we found that 3 reacts with $\mathrm{BuCl}$ in $89 \%$ yield within $13 \mathrm{~h}$. A prolongation of the reaction time does not lead to a significant improvement in yield. Thus, the quaternization 
E $\mathrm{C}_{n}$ gBF4 目 $\mathrm{C}_{n}$-gSac $2 \mathrm{C}_{n}$-gAce $\Delta \mathrm{C}_{n}$ gTos

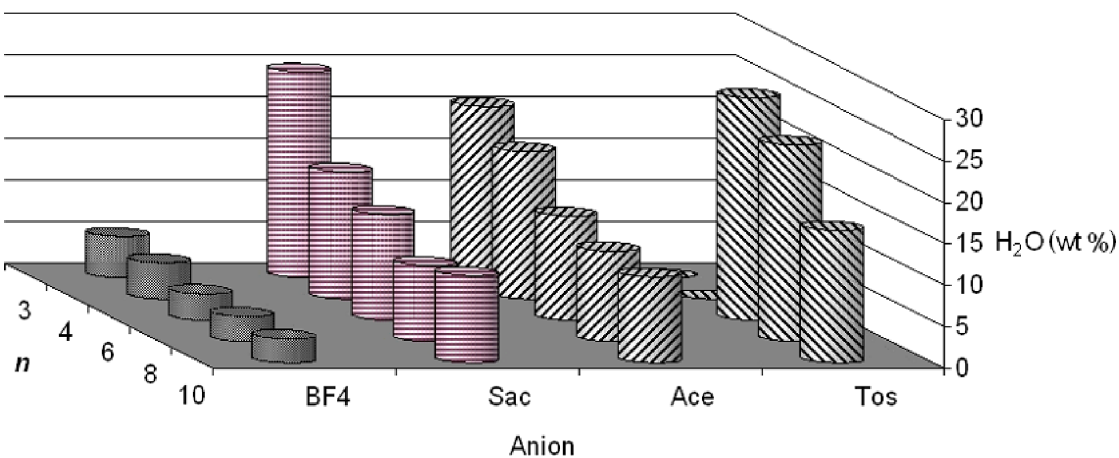

Fig. 1. Solubility of water in $\mathbf{C}_{\boldsymbol{n}}$-gILs as a function of the nature of the anions and the number of carbon atoms in the alkyl chain $n$ at room temperature. reactions of guanidine 3 with butyl, hexyl, octyl, and decyl chloride on a larger scale $(0.1 \mathrm{~mol})$ were carried out with a threefold molar excess of the corresponding alkyl chloride at $100-110{ }^{\circ} \mathrm{C}$ in DMF as solvent for approximately $13 \mathrm{~h}$. IR spectroscopy proved to be a good method for monitoring the reaction in this case, since the disappearance of the $\mathrm{C}=\mathrm{N}$ band of $\mathbf{3}$ at about $1610 \mathrm{~cm}^{-1}$ and the appearance of a $\mathrm{C}^{+}-\mathrm{N}$ band of $\mathbf{C}_{\boldsymbol{n}}$-gCl at about $1530 \mathrm{~cm}^{-1}$ can be easily observed. Thus, $\mathbf{C}_{n}$-gCl $(n=4,6,8,10)$ were obtained in 80 $90 \%$ yields. It should be mentioned that the successful progress of the reaction is limited to a certain temperature range. A reaction temperature above $110{ }^{\circ} \mathrm{C}$ results in a reversion of the quaternization reaction (detected by IR spectroscopy), and the use of temperatures lower than $80^{\circ} \mathrm{C}$ results in significant prolongation of the reaction time, and thus in lowering the yields. Limited by the boiling point of propyl chloride (b. p. $46.7^{\circ} \mathrm{C}$ ), the quaternization reaction in this case was carried out at $60{ }^{\circ} \mathrm{C}$ and with a greater excess of the latter (added portionwise), leading to the formation of $\mathbf{C}_{\mathbf{3}}-\mathbf{g C l}$ in $30 \%$ yield.

As can be seen from Table 2, all of the synthesized hexaalkylguanidinium chlorides are miscible with water and immiscible with diethyl ether or cyclohexane. This allows their isolation and purification by dissolving each product in a small quantity of water and subsequent extraction of the residual volatile organic components. This approach was very useful, especially in the case of high-boiling alkyl chlorides. Except for $\mathbf{C}_{6}$ gCl, which crystallized spontaneously after drying under vacuum, the other guanidinium chlorides $\mathbf{C}_{\boldsymbol{n}}$-gCl $(n=3,4,8,10)$ are liquids at room temperature.

The synthesis of tetrafluoroborate $\left(\mathrm{BF}_{4}\right)$, acesulfamate (Ace) and saccharinate (Sac) gILs was achieved by metathesis reactions of $\mathbf{C}_{\boldsymbol{n}}$-gCl with the correspond- ing sodium or potassium salt in methanol or water as solvents. The tosylate series was synthesized by alkylation of guanidine $\mathbf{3}$ with the corresponding tosylate. The reactions were carried out under the quaternization conditions described above, but with a smaller excess (1.1 equiv.) of the tosyl ester. In all cases the reactions proceeded quantitatively within $4.5 \mathrm{~h}$. All synthesized compounds were characterized by spectroscopic methods $\left({ }^{1} \mathrm{H},{ }^{13} \mathrm{C}\right.$ NMR, IR), and the purity was established by elemental analysis. Furthermore, some properties of the synthesized gILs, such as miscibility with organic solvents, water uptake ability and density, were assessed as a function of the anion and of the chain length of the alkyl substituent.

\section{Properties}

Table 2 shows molecular weights, densities, water content, water uptake ability, and solubility in solvents of the synthesized RTgILs. For water-insoluble gILs, the water content prior to the property measurement was estimated (by Karl-Fischer titration) to be in the range 300 to $550 \mathrm{ppm}$ and between 600 and $750 \mathrm{ppm}$ for the water soluble $\mathbf{C}_{\boldsymbol{n}}$-gCl $(n=3,4,6,8,10)$ and $\mathbf{C}_{\boldsymbol{n}}$-gTos $(n=1,2,4)$. It is noteworthy that all $\mathbf{C}_{\boldsymbol{n}}$-gILs are strongly hygroscopic. The capacity to absorb water from the air was found to depend of course on the relative atmospheric humidity, but also on the length of the alkyl substituent $\mathrm{R}$. The absorbed water is readily released by heating under vacuum, and thus, the synthesized RTgILs might be considered as desiccants.

The compounds studied are, however, stable in the air and in common organic solvents such as methanol, acetone, dichloromethane, chloroform, and acetonitrile, in which they are completely soluble. Furthermore, they are insoluble (formation of two phases is 
observed) in cyclohexane, diethyl ether and water, but their miscibility with the latter depends not only on the hydrophobicity of the cation, but also on the nature of the anion and the temperature. These exceptional solubility characteristics in general make them good candidates for multiphasic reactions, liquid extraction and separation technology $[14,15]$. To examine the affinity for the absorption of water, equivalent quantities of the corresponding hydrophobic gIL and water were shaken vigorously in a closed tube, and then the water content in the IL layer was estimated by Karl-Fischer titration. Fig. 1 presents the water content of the water-saturated $\mathbf{C}_{\boldsymbol{n}}$-gILs in wt- $\%$. The observed decrease in water uptake ability with increasing number $n$ of the carbon atoms in the alkyl chain clearly shows that the alkyl chain length corresponds to the hydrophobicity. On the other hand, the nature of the anion controls the hydrophobicity as well. Regarding the influence of the anion, the water uptake ability decreases as follows: $\mathbf{C}_{\boldsymbol{n}}$-gTos $>\mathbf{C}_{\boldsymbol{n}}$-gAce $\approx \mathbf{C}_{\boldsymbol{n}^{-}}$$\mathbf{g S a c}>\mathbf{C}_{\boldsymbol{n}}-\mathbf{g B F}_{\mathbf{4}}$. This sequence can be rationalized by taking into account the basicity of the corresponding anions. Furthermore, three immiscible liquid layers were obtained by mixing water, cyclohexane or diethyl ether, and selected gILs. The formation of a sequence water/gIL/diethyl ether depends clearly on the density of the ILs under study. Thus, the less dense hydrophobic gILs are positioned between the water and the organic phases forming a third one. On the one hand, this is due to the lower density value of the IL under study compared to water, and on the other hand, due to the partial miscibility of diethyl ether with the IL which results in a further reduction of the density. Although there are many examples of solubilization of organic solvents in hydrophobic ILs, most of the hydrophobic ILs are of high density (around $1.4 \mathrm{~g} \mathrm{~cm}^{-3}$ ) [15], and the density never is below $1.0 \mathrm{~g} \mathrm{~cm}^{-3}$. Thus, the behavior in layer sequence shown here is unique for the synthesized relatively "light" gILs and might be of interest in novel reactions or in separation processes or devices.

It has recently been reported that the density of some guanidinium-based RTILs is relatively low compared to the imidazolium series [4b], and that the guanidinium ion is more capable to suppress the effect of the anion than the other cations. In this direction, we found in the present study the same trend in the density behavior, namely, the densities of all of the five series under study are in the range 0.989$1.069 \mathrm{~g} \mathrm{~cm}^{-3}$, regardless of the anion. Considering the cation, the density decreases as the number of carbon atoms $n$ in the guanidinium ion $\mathbf{C}_{\boldsymbol{n}}-\mathbf{g}^{+}$increases, which is typical for RTILs. Thus, the higher the mass of the cation, the lower the density is in a given series (see Table 2). Furthermore, the change of the butyl substituent to decyl in the guanidinium ion leads to a decrease in density for the tertafluoroborate series $\mathbf{C}_{\boldsymbol{n}}-\mathbf{g B F}_{\mathbf{4}}$ from 1.045 to $0.981 \mathrm{~g} \mathrm{~cm}^{-3}$, which is rather negligible compared to the methylimidazolium series $\mathbf{C}_{\boldsymbol{n}}$-mimBF , with changes from 1.208 to 1.072 for the same substituents. Considering the anion, when the cation is kept constant, the order of density decrease is as follows: $\mathrm{Sac}^{-}>\mathrm{Ace}^{-}>\mathrm{Tos}^{-}>\mathrm{BF}_{4}{ }^{-}$, but just as in the case of the cation, the effect of the anion is negligible, and thus, the densities for the $\mathbf{C}_{\mathbf{4}} \mathbf{g}^{+}$series are: $1.063 \approx 1.050 \approx 1.027 \approx 1.009$, following the anion sequence considered above. These effects might be attributed to the higher volume (mass respectively) of the guanidinium ion compared to the volume of the counterions. Consequently, it might be concluded that the density of a RTgIL can be determined by using the appropriate guanidinium ion, and at the same time other desirable properties may be varied by simply changing the substituents or counterions. This can be of a great importance for the industrial application of RTILs.

As we mentioned above, the density values of the gILs under study seem to be determined by the molecular volume (mass) of the guanidinium ion. In spite of the negligible differences in density in the separate series, we found this property to be further fine-tunable by using the Residual Volume Approach (RVA), a recently developed method for predicting some physical properties of ILs by using simple correlations between the desired property and newly defined substituent parameters $\beta^{\mathrm{X}}[16,17]$. Plots of $\rho=f(\beta)$ for the synthesized RTgILs are shown in Fig. 2. As can be seen, the density decreases with $\beta^{\mathrm{X}}$, and each series follows a very good linear relationship $\left(R^{2}=0.97-0.99\right)$. The results obtained from Fig. 2 show that the RVA can be applied successfully in the case of guanidinium-based ILs as well, and can be used for predicting the density values of the remaining members of the series by using the empirically obtained linear equation for a given series and the substituent constants $\beta^{\mathrm{X}}[16,17]$.

\section{Conclusion}

Several series of new homologous $N^{\prime \prime}$-n-alkylsubstituted $N, N$-diethyl- $N^{\prime}, N^{\prime}$-di- $n$-propyl- $N^{\prime \prime}$ - $n$-hexyl guanidinium salts with tetrafluoroborate, acesulfamate, saccharinate, and tosylate anions were synthesized and 


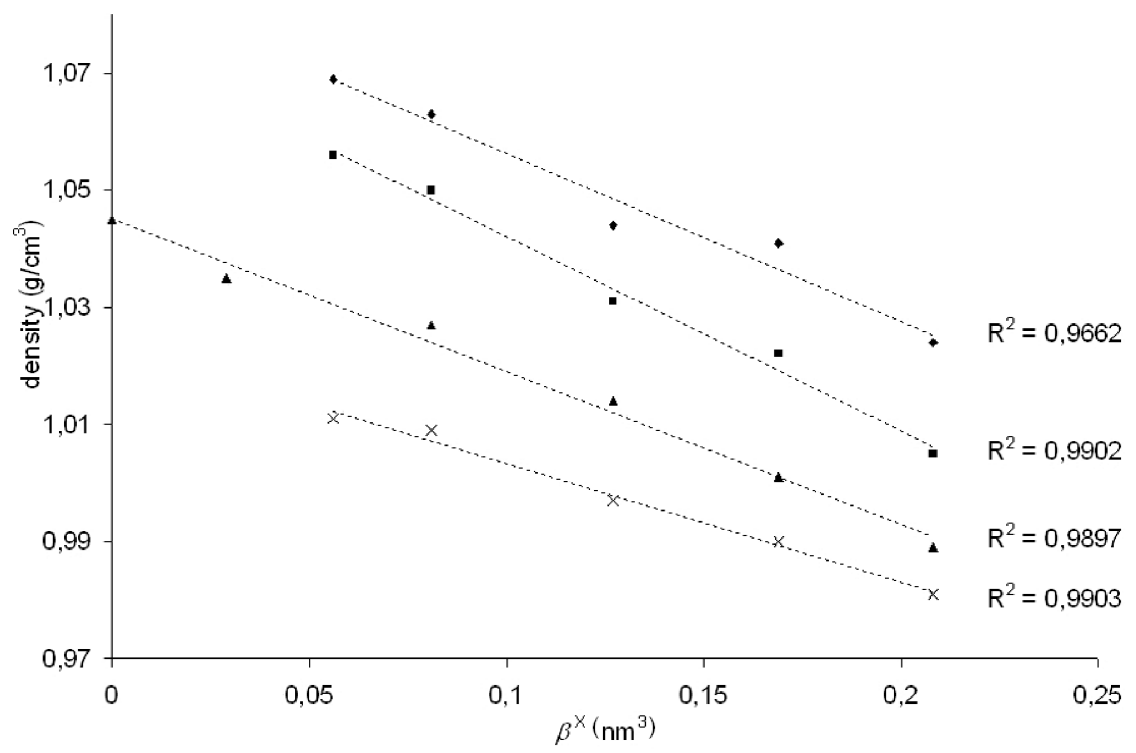

Fig. 2. Residual Volume Approach correlations of densities at $25{ }^{\circ} \mathrm{C}:-\mathrm{C}_{n^{-}} \mathrm{gSac}, \boldsymbol{\mathrm { C } _ { n ^ { - } }}$ gAce, $\boldsymbol{\Delta} \mathrm{C}_{n}$-gTos, $n=1,2,4$, $6,8,10$ and $\times \mathrm{C}_{n}-\mathrm{gBF}_{4}, n=3$, $4,6,8,10$. characterized and their physical properties were evaluated. All the salts are hydrophobic liquids at room temperature, allowing an investigation of their water uptake ability and density. The behavior is unique for the synthesized relatively "light" hydrophobic gILs, which might be of interest in novel reactions or in separation processes and devices.

\section{Experimental Section}

\section{General remarks}

Commercially available reagents were used as supplied. All the other reagents used were dried and freshly distilled prior to use. Distilled water was used for the preparation of aqueous solutions. All glassware was oven-dried at $75^{\circ} \mathrm{C}$ before using.

The IR spectra were acquired from thin films on a PerkinElmer FT-IR instrument Model Spectrum One and are reported in reciprocal centimeters. The ${ }^{1} \mathrm{H}$ and ${ }^{13} \mathrm{C}$ NMR spectra were obtained on a Bruker Avance DRX-250 NMR spectrometer operating at 250.13 and $62.9 \mathrm{~Hz}$, respectively, in the solvent given in parentheses. The chemical shifts are given in $\operatorname{ppm}(\delta)$ relative to tetramethylsilane as internal standard. Elemental analyses were obtained in the laboratories at the Institute of Organic Chemistry, University of Stuttgart. Prior to their further manipulation (characterization or property measurement), each RTIL was dried under vacuum (0.01 mbar) at $90{ }^{\circ} \mathrm{C}$ for at least $6 \mathrm{~h}$.

\section{Water contents}

The water content of each RTgIL was determined by KarlFischer titration with a Metrohm 831 KF Coulometer.
Density

The reported densities were determined by means of a picnometer $\left(1 \mathrm{~cm}^{3}\right)$ at $25^{\circ} \mathrm{C}$. The mass of the volume of liquid was taken on an analytical balance and each reported value is the average of two measurements.

\section{Synthesis}

$N, N$-diethyl- $N^{\prime}, N^{\prime}$-di- $n$-propylurea (1), $N, N$-diethyl- $N^{\prime}$, $N^{\prime}$-di- $n$-propylchloroformamidinium chloride (2) and the pentasubstituted guanidine $\mathbf{3}$ were synthesized by protocols published previously by Kantlehner and coworkers [2].

General procedure for the synthesis of guanidinium chlorides $\boldsymbol{C}_{\boldsymbol{n}}-\boldsymbol{g C l}(n=3,4,6,8,10)$

The reactions were carried out in round-bottom flasks equipped with a reflux condenser and a calcium chloride tube. The corresponding alkyl chlorides (3 equiv.) were added to a solution of guanidine $\mathbf{3}$ in dry DMF, and the reaction mixtures were stirred at $100-110{ }^{\circ} \mathrm{C}$ (oil bath) for an appropriate time. The consumption of $\mathbf{3}$ was monitored by FTIR. After the end of the reaction the volatile ingredients were evaporated under reduced pressure, the residue was dissolved in distilled water and in order to remove any unreacted starting material washed with cyclohexane $\left(6 \times 50 \mathrm{~cm}^{3}\right)$. Afterwards, the main quantity of water was evaporated under reduced pressure $\left(90^{\circ} \mathrm{C}, 20 \mathrm{mbar}\right.$ ), and the residual RTILs $\mathbf{C}_{\boldsymbol{n}}$ gCl were further dried at high vacuum $\left(90^{\circ} \mathrm{C}, 0.01 \mathrm{mbar}\right)$ for 6-8 h. Except $\mathbf{C}_{\mathbf{6}}-\mathbf{g C l}$, which solidified after drying, all the other guanidinium chlorides were obtained as slightly yellow liquids. 
$N, N$-Diethyl- $N^{\prime}, N^{\prime}$-di-n-propyl- $N^{\prime \prime}-n$-hexyl- $N^{\prime \prime}-n$ propylguanidinium chloride $\left(\boldsymbol{C}_{\mathbf{3}}-\mathbf{g} \boldsymbol{C l}\right)$

This compound was obtained from $43.98 \mathrm{~g}(0.56 \mathrm{~mol})$ of $n$-propyl chloride and $39.66 \mathrm{~g}(0.14 \mathrm{~mol})$ of guanidine 3. - Yield $15.21 \mathrm{~g}(30 \%)$. - IR (film): $v=1533$ $\left(\mathrm{CN}_{3}{ }^{+}\right) \mathrm{cm}^{-1}$. - ${ }^{1} \mathrm{H}$ NMR $\left(250.13 \mathrm{MHz}, \mathrm{CDCl}_{3}\right): \delta=$ $0.78-1.02\left(\mathrm{~m}, 12 \mathrm{H}, 3 \times \mathrm{C}_{2} \mathrm{H}_{4} \mathrm{CH}_{3}, \mathrm{C}_{5} \mathrm{H}_{10} \mathrm{CH}_{3}\right), 1.14-1.35$ $\left(\mathrm{m}, 12 \mathrm{H}, 3 \times \mathrm{CH}_{2}, 2 \times \mathrm{CH}_{2} \mathrm{CH}_{3}\right), 1.38-1.95(\mathrm{~m}, 8 \mathrm{H}$, $\left.\mathrm{NCH}_{2} \mathrm{CH}_{2}, 3 \times \mathrm{NCH}_{2} \mathrm{CH}_{2} \mathrm{CH}_{3}\right), 2.96-3.55(\mathrm{~m}, 12 \mathrm{H}$, all $\left.\mathrm{CH}_{2} \mathrm{~N}\right) .-{ }^{13} \mathrm{C} \mathrm{NMR}\left(62.9 \mathrm{MHz}, \mathrm{CDCl}_{3}\right): \delta=11.3(2 \times$ $\left.\mathrm{CH}_{3}\right), 11.4\left(\mathrm{CH}_{3}\right), 13.0\left(\mathrm{CH}_{3}\right), 13.1\left(\mathrm{CH}_{3}\right), 13.7\left(\mathrm{CH}_{3}\right), 21.0$ $\left(\mathrm{CH}_{2}\right), 22.5\left(3 \times \mathrm{CH}_{2}\right), 26.8\left(\mathrm{CH}_{2}\right), 27.5\left(\mathrm{CH}_{2}\right), 31.1\left(\mathrm{CH}_{2}\right)$, $44.1\left(2 \times \mathrm{CH}_{2} \mathrm{~N}\right), 49.8\left(\mathrm{CH}_{2} \mathrm{~N}\right), 51.4\left(2 \times \mathrm{CH}_{2} \mathrm{~N}\right), 51.5$ $\left(\mathrm{CH}_{2} \mathrm{~N}\right), 163.9\left(\mathrm{CN}_{3}{ }^{+}\right)$.

$N, N$-Diethyl- $N^{\prime}, N^{\prime}$-di-n-propyl- $N^{\prime \prime}-n$-hexyl- $N^{\prime \prime}-n$ butylguanidinium chloride $\left(\boldsymbol{C}_{\mathbf{4}}-\mathbf{g} \boldsymbol{C l}\right)$

This compound was obtained from $58.55 \mathrm{~cm}^{3}(51.52 \mathrm{~g}$, $0.56 \mathrm{~mol})$ of $n$-butyl chloride and $39.66 \mathrm{~g}(0.14 \mathrm{~mol})$ of guanidine 3. - Yield $44.63 \mathrm{~g}(89 \%) .-n_{\mathrm{D}}^{20}=1.5007$. - IR (film): $v=1532\left(\mathrm{CN}_{3}{ }^{+}\right) \mathrm{cm}^{-1} .-{ }^{1} \mathrm{H}$ NMR $(250.13 \mathrm{MHz}$, $\left.\mathrm{CDCl}_{3}\right): \delta=0.80-1.03\left(\mathrm{~m}, 12 \mathrm{H}, 2 \times \mathrm{C}_{2} \mathrm{H}_{4} \mathrm{CH}_{3}, \mathrm{C}_{3} \mathrm{H}_{6} \mathrm{CH}_{3}\right.$, $\left.\mathrm{C}_{5} \mathrm{H}_{10} \mathrm{CH}_{3}\right), 1.12-1.95\left(\mathrm{~m}, 22 \mathrm{H}, 8 \times \mathrm{CH}_{2}, 2 \times \mathrm{CH}_{2} \mathrm{CH}_{3}\right)$, $2.92-3.56\left(\mathrm{~m}, 12 \mathrm{H}\right.$, all $\left.\mathrm{CH}_{2} \mathrm{~N}\right) .-{ }^{13} \mathrm{C}$ NMR $(62.9 \mathrm{MHz}$, $\left.\mathrm{CDCl}_{3}\right): \delta=11.3\left(\mathrm{CH}_{3}\right), 11.4\left(\mathrm{CH}_{3}\right), 13.1\left(\mathrm{CH}_{3}\right), 13.7$ $\left(\mathrm{CH}_{3}\right), 13.9\left(2 \times \mathrm{CH}_{3}\right), 20.1\left(\mathrm{CH}_{2}\right), 21.0\left(\mathrm{CH}_{2}\right), 22.5(2 \times$ $\left.\mathrm{CH}_{2}\right), 26.6\left(\mathrm{CH}_{2}\right), 27.4\left(\mathrm{CH}_{2}\right), 29.6\left(\mathrm{CH}_{2}\right), 31.3\left(\mathrm{CH}_{2}\right), 44.2$ $\left(2 \times \mathrm{CH}_{2} \mathrm{~N}\right), 49.6\left(\mathrm{CH}_{2} \mathrm{~N}\right), 49.8\left(\mathrm{CH}_{2} \mathrm{~N}\right), 51.5\left(2 \times \mathrm{CH}_{2} \mathrm{~N}\right)$, $163.9\left(\mathrm{CN}_{3}{ }^{+}\right)$.

$N, N$-Diethyl- $N^{\prime}, N^{\prime}$-di-n-propyl- $N^{\prime \prime}, N^{\prime \prime}-d i-n$ hexylguanidinium chloride $\left(\boldsymbol{C}_{\mathbf{6}}-\mathbf{g} \boldsymbol{C l}\right)$

This compound was obtained from $21.2 \mathrm{~cm}^{3}(23.88 \mathrm{~g}$, $0.20 \mathrm{~mol})$ of $n$-hexyl chloride and $14.0 \mathrm{~g}(0.05 \mathrm{~mol})$ of guanidine 3. - Yield $17.4 \mathrm{~g}(87 \%) .-n_{\mathrm{D}}^{20}=1.4934$. - IR (film): $v=1532\left(\mathrm{CN}_{3}{ }^{+}\right) \mathrm{cm}^{-1} .-{ }^{1} \mathrm{H} \mathrm{NMR}\left(250.13 \mathrm{MHz}, \mathrm{CDCl}_{3}\right)$ : $\delta=0.79-1.02\left(\mathrm{~m}, 12 \mathrm{H}, 2 \times \mathrm{C}_{2} \mathrm{H}_{4} \mathrm{CH}_{3}, 2 \times \mathrm{C}_{5} \mathrm{H}_{10} \mathrm{CH}_{3}\right)$, $1.16-1.95\left(\mathrm{~m}, 26 \mathrm{H}, 10 \times \mathrm{CH}_{2}, 2 \times \mathrm{CH}_{2} \mathrm{CH}_{3}\right), 2.96-3.57$ $\left(\mathrm{m}, 12 \mathrm{H}\right.$, all $\left.\mathrm{CH}_{2} \mathrm{~N}\right) \cdot-{ }^{13} \mathrm{C} \mathrm{NMR}\left(62.9 \mathrm{MHz}, \mathrm{CDCl}_{3}\right): \delta=$ $11.3\left(\mathrm{CH}_{3}\right), 11.4\left(\mathrm{CH}_{3}\right), 13.1\left(2 \times \mathrm{CH}_{3}\right), 13.9\left(2 \times \mathrm{CH}_{3}\right)$, $21.0\left(\mathrm{CH}_{2}\right), 21.1\left(\mathrm{CH}_{2}\right), 22.5\left(2 \times \mathrm{CH}_{2}\right), 26.5\left(\mathrm{CH}_{2}\right), 26.6$ $\left(\mathrm{CH}_{2}\right), 27.4\left(\mathrm{CH}_{2}\right), 27.6\left(\mathrm{CH}_{2}\right), 31.3\left(2 \times \mathrm{CH}_{2}\right), 44.2(2 \times$ $\left.\mathrm{CH}_{2} \mathrm{~N}\right), 49.8\left(2 \times \mathrm{CH}_{2} \mathrm{~N}\right), 51.5\left(\mathrm{CH}_{2} \mathrm{~N}\right), 51.6\left(\mathrm{CH}_{2} \mathrm{~N}\right), 163.9$ $\left(\mathrm{CN}_{3}{ }^{+}\right)$.

$N, N$-Diethyl- $N^{\prime}, N^{\prime}$-di-n-propyl- $N^{\prime \prime}-n$-hexyl- $N^{\prime \prime}-n$ octylguanidinium chloride $\left(\boldsymbol{C}_{\mathbf{8}} \mathbf{- g} \boldsymbol{C l}\right)$

This compound was obtained from $47.5 \mathrm{~cm}^{3}$ (41.47 g, $0.28 \mathrm{~mol})$ of $n$-octyl chloride and $19.7 \mathrm{~g}(0.07 \mathrm{~mol})$ of guanidine 3. - Yield $25.4 \mathrm{~g}(85 \%) .-n_{\mathrm{D}}^{20}=1.4957$. - IR (film): $v=1533\left(\mathrm{CN}_{3}{ }^{+}\right) \mathrm{cm}^{-1} .-{ }^{1} \mathrm{H} \mathrm{NMR}\left(250.13 \mathrm{MHz}, \mathrm{CDCl}_{3}\right)$ : $\delta=0.79-1.03\left(\mathrm{~m}, 12 \mathrm{H}, 2 \times \mathrm{C}_{2} \mathrm{H}_{4} \mathrm{CH}_{3}, \mathrm{C}_{5} \mathrm{H}_{10} \mathrm{CH}_{3}\right.$, $\left.\mathrm{C}_{7} \mathrm{H}_{14} \mathrm{CH}_{3}\right), 1.11-1.90\left(\mathrm{~m}, 30 \mathrm{H}, 12 \times \mathrm{CH}_{2}, 2 \times \mathrm{CH}_{2} \mathrm{CH}_{3}\right)$, 2.97-3.64 (m, $12 \mathrm{H}$, all $\left.\mathrm{CH}_{2} \mathrm{~N}\right) .-{ }^{13} \mathrm{C}$ NMR $(62.9 \mathrm{MHz}$, $\left.\mathrm{CDCl}_{3}\right): \delta=11.3\left(\mathrm{CH}_{3}\right), 11.4\left(\mathrm{CH}_{3}\right), 13.1\left(\mathrm{CH}_{3}\right), 13.2$ $\left(\mathrm{CH}_{3}\right), 13.9\left(\mathrm{CH}_{3}\right), 14.0\left(\mathrm{CH}_{3}\right), 21.0\left(\mathrm{CH}_{2}\right), 21.1\left(\mathrm{CH}_{2}\right)$, $22.6\left(2 \times \mathrm{CH}_{2}\right), 26.6\left(\mathrm{CH}_{2}\right), 26.9\left(\mathrm{CH}_{2}\right), 27.5\left(2 \times \mathrm{CH}_{2}\right)$, $29.1\left(2 \times \mathrm{CH}_{2}\right), 31.3\left(\mathrm{CH}_{2}\right), 31.7\left(\mathrm{CH}_{2}\right), 44.2\left(\mathrm{CH}_{2} \mathrm{~N}\right)$, $44.3\left(\mathrm{CH}_{2} \mathrm{~N}\right), 49.8\left(2 \times \mathrm{CH}_{2} \mathrm{~N}\right), 51.5\left(\mathrm{CH}_{2} \mathrm{~N}\right), 51.6\left(\mathrm{CH}_{2} \mathrm{~N}\right)$, $164.0\left(\mathrm{CN}_{3}{ }^{+}\right)$.

\section{$N, N$-Diethyl- $N^{\prime}, N^{\prime}$-di-n-propyl- $N^{\prime \prime}-n$-hexyl- $N^{\prime \prime}-n$ - decylguanidinium chloride $\left(\boldsymbol{C}_{\mathbf{1 0}}-\mathbf{g C l}\right)$}

This compound was obtained from $38.05 \mathrm{~cm}^{3}(33.11 \mathrm{~g}$, $0.188 \mathrm{~mol})$ of $n$-decyl chloride and $13.28 \mathrm{~g}(0.047 \mathrm{~mol})$ of guanidine 3. - Yield $51.4 \mathrm{~g}(74 \%) .-n_{\mathrm{D}}^{20}=1.4935$. $-\mathrm{IR}$ (film): $v=1534\left(\mathrm{CN}_{3}{ }^{+}\right) \mathrm{cm}^{-1}$. - ${ }^{1} \mathrm{H}$ NMR $(250.13 \mathrm{MHz}$, $\left.\mathrm{CDCl}_{3}\right): \delta=0.81-1.02\left(\mathrm{~m}, 12 \mathrm{H}, 2 \times \mathrm{C}_{2} \mathrm{H}_{4} \mathrm{CH}_{3}\right.$, $\left.\mathrm{C}_{5} \mathrm{H}_{10} \mathrm{CH}_{3}, \mathrm{C}_{9} \mathrm{H}_{18} \mathrm{CH}_{3}\right), 1.07-1.94\left(\mathrm{~m}, 34 \mathrm{H}, 14 \times \mathrm{CH}_{2}\right.$, $\left.2 \times \mathrm{CH}_{2} \mathrm{CH}_{3}\right), 3.00-3.64\left(\mathrm{~m}, 12 \mathrm{H}\right.$, all $\left.\mathrm{CH}_{2} \mathrm{~N}\right) .-{ }^{13} \mathrm{C} \mathrm{NMR}$ $\left(62.9 \mathrm{MHz}, \mathrm{CDCl}_{3}\right): \delta=11.3\left(\mathrm{CH}_{3}\right), 11.4\left(\mathrm{CH}_{3}\right), 13.1(2 \times$ $\left.\mathrm{CH}_{3}\right), 13.9\left(\mathrm{CH}_{3}\right), 14.1\left(\mathrm{CH}_{3}\right), 21.0\left(\mathrm{CH}_{2}\right), 21.1\left(\mathrm{CH}_{2}\right), 22.5$ $\left(\mathrm{CH}_{2}\right), 22.6\left(\mathrm{CH}_{2}\right), 26.9\left(\mathrm{CH}_{2}\right), 27.0\left(\mathrm{CH}_{2}\right), 27.4\left(2 \times \mathrm{CH}_{2}\right)$, $29.2\left(2 \times \mathrm{CH}_{2}\right), 29.4\left(2 \times \mathrm{CH}_{2}\right), 31.3\left(\mathrm{CH}_{2}\right), 31.8\left(\mathrm{CH}_{2}\right)$, $44.2\left(\mathrm{CH}_{2} \mathrm{~N}\right), 44.3\left(\mathrm{CH}_{2} \mathrm{~N}\right), 49.8\left(2 \times \mathrm{CH}_{2} \mathrm{~N}\right), 51.5\left(\mathrm{CH}_{2} \mathrm{~N}\right)$, $51.6\left(\mathrm{CH}_{2} \mathrm{~N}\right), 163.9\left(\mathrm{CN}_{3}{ }^{+}\right)$.

General procedure for the synthesis of guanidinium p-toluenesulfonates $\boldsymbol{C}_{\boldsymbol{n}}$-gTos $(n=1,2,4,6,8,10)$

In a round-bottom flask equipped with a reflux condenser, the pentasubstituted guanidine $\mathbf{3}$ was added to a stirred solution of the corresponding tosylate ester in dry DMF. The reaction mixture was then stirred at $110{ }^{\circ} \mathrm{C}$ for $2-4.30 \mathrm{~h}$. The reaction was monitored by IR spectroscopy. At the end of the reaction the volatile compounds were evaporated on a rotary evaporator, and the residue was washed with diethyl ether $\left(5 \times 50 \mathrm{~cm}^{3}\right)$. The residue was further evaporated first at $90{ }^{\circ} \mathrm{C} / 23 \mathrm{mbar}$, and than at $0.01 \mathrm{mbar}$ for $8 \mathrm{~h}$.

\section{$N, N$-Diethyl- $N^{\prime}, N^{\prime}$-di-n-propyl- $N^{\prime \prime}$-n-hexyl- $N^{\prime \prime}$ -} methylguanidinium p-toluenesulfonate $\left(\boldsymbol{C}_{\mathbf{1}}-\mathbf{g T o s}\right)$

This compound was obtained from $11.9 \mathrm{~g}(0.0639 \mathrm{~mol})$ of methyl tosylate and $16.5 \mathrm{~g}(0.0581 \mathrm{~mol})$ of guanidine $\mathbf{3}$ in DMF $\left(110 \mathrm{~cm}^{3}\right)$. - Yield: $27.02 \mathrm{~g}(99 \%) .-n_{\mathrm{D}}^{20}=1.5145$. IR (film): $v=1539\left(\mathrm{CN}_{3}^{+}\right), 1200\left(\mathrm{SO}_{3}^{-}\right), 677(\mathrm{Ar}) \mathrm{cm}^{-1}$. ${ }^{1} \mathrm{H}$ NMR $\left(250.13 \mathrm{MHz}, \mathrm{CDCl}_{3}\right): \delta=0.75-1.02(\mathrm{~m}, 9 \mathrm{H}$, $\left.2 \times \mathrm{C}_{2} \mathrm{H}_{4} \mathrm{CH}_{3}, \mathrm{C}_{5} \mathrm{H}_{10} \mathrm{CH}_{3}\right), 1.09-1.84\left(\mathrm{~m}, 18 \mathrm{H}, 6 \times \mathrm{CH}_{2}\right.$, $\left.2 \times \mathrm{CH}_{2} \mathrm{CH}_{3}\right), 2.32\left(\mathrm{~s}, 3 \mathrm{H}, \mathrm{CH}_{3}-\mathrm{C}_{6} \mathrm{H}_{4} \mathrm{SO}_{3}{ }^{-}\right), 2.86-3.56$ $\left(\mathrm{m}, 10 \mathrm{H}\right.$, all $\left.\mathrm{CH}_{2} \mathrm{~N}\right), 3.03\left(\mathrm{~d}, \mathrm{~J}=3.1 \mathrm{~Hz}, 3 \mathrm{H}, \mathrm{NCH}_{3}\right), 7.10$ $\left(\mathrm{d}, J=7.9 \mathrm{~Hz}, 2 \mathrm{H}, \mathrm{CH}_{3} C_{6} \mathrm{H}_{4} \mathrm{SO}_{3}{ }^{-}\right), 7.79(\mathrm{~d}, J=8.1 \mathrm{~Hz}$, $\left.2 \mathrm{H}, \mathrm{CH}_{3} \mathrm{C}_{6} \mathrm{H}_{4} \mathrm{SO}_{3}{ }^{-}\right) .-{ }^{13} \mathrm{C} \mathrm{NMR}\left(62.9 \mathrm{MHz}, \mathrm{CDCl}_{3}\right)$ : $\delta=11.4\left(\mathrm{CH}_{3}\right), 12.7\left(\mathrm{CH}_{3}\right), 12.9\left(\mathrm{CH}_{3}\right), 13.1\left(\mathrm{CH}_{3}\right), 13.9$ 
$\left(\mathrm{CH}_{3}\right), 20.8\left(\mathrm{CH}_{2}\right), 21.0\left(\mathrm{CH}_{2}\right), 21.3\left(\mathrm{CH}_{3}\right), 22.5\left(\mathrm{CH}_{2}\right)$, $26.5\left(\mathrm{CH}_{2}\right), 27.2\left(\mathrm{CH}_{2}\right), 31.3\left(\mathrm{CH}_{2}\right), 38.6$ and $38.9\left(\mathrm{CH}_{3} \mathrm{~N}-\right.$ rotamers $), 43.3\left(\mathrm{CH}_{2} \mathrm{~N}\right), 43.7\left(\mathrm{CH}_{2} \mathrm{~N}\right), 44.0\left(\mathrm{CH}_{2} \mathrm{~N}\right), 51.3$ $\left(\mathrm{CH}_{2} \mathrm{~N}\right), 52.9\left(\mathrm{CH}_{2} \mathrm{~N}\right), 126.1(2 \times-\mathrm{CH}=), 128.3(2 \times-$ $\mathrm{CH}=), 138.5\left(\mathrm{C}-\mathrm{CH}_{3}\right), 144.5\left(\mathrm{C}-\mathrm{SO}_{3}\right), 164.0\left(\mathrm{CN}_{3}{ }^{+}\right) .-$ Anal. for $\mathrm{C}_{25} \mathrm{H}_{47} \mathrm{~N}_{3} \mathrm{O}_{3} \mathrm{~S}(469.7) \times \mathrm{H}_{2} \mathrm{O}$ : calcd. C 61.56, H 10.13, N 8.62; found C 61.55, H 9.95, N 8.53.

$N, N$-Diethyl- $N^{\prime}, N^{\prime}$-di-n-propyl- $N^{\prime \prime}-n$-hexyl- $N^{\prime \prime}$ ethylguanidinium p-toluenesulfonate $\left(\boldsymbol{C}_{2}-\mathbf{g T o s}\right)$

This compound was obtained from $8.58 \mathrm{~g}(0.0428 \mathrm{~mol})$ of ethyl tosylate and $11.04 \mathrm{~g}(0.0390 \mathrm{~mol})$ of guanidine $\mathbf{3}$ in DMF $\left(80 \mathrm{~cm}^{3}\right)$. - Yield: $17.7 \mathrm{~g}(94 \%) .-n_{\mathrm{D}}^{20}=1.5136$. IR (film): $v=1535\left(\mathrm{CN}_{3}{ }^{+}\right), 1201\left(\mathrm{SO}_{3}^{-}\right), 678$ (Ar). $\left.{ }^{1} \mathrm{H} \mathrm{NMR} \mathrm{(250.13} \mathrm{MHz,} \mathrm{CDCl}_{3}\right): \delta=0.74-1.02(\mathrm{~m}, 9 \mathrm{H}, 2 \times$ $\left.\mathrm{C}_{2} \mathrm{H}_{4} \mathrm{CH}_{3}, \mathrm{C}_{5} \mathrm{H}_{10} \mathrm{CH}_{3}\right), 1.05-1.88\left(\mathrm{~m}, 21 \mathrm{H}, 6 \times \mathrm{CH}_{2}, 3 \times\right.$ $\mathrm{CH}_{2} \mathrm{CH}_{3}$ ), 2.32 (s, $3 \mathrm{H}, \mathrm{CH}_{3}-\mathrm{C}_{6} \mathrm{H}_{4} \mathrm{SO}_{3}^{-}$), $2.87-3.56$ (m, $12 \mathrm{H}$, all $\left.\mathrm{CH}_{2} \mathrm{~N}\right), 7.10\left(\mathrm{~d}, J=7.9 \mathrm{~Hz}, 2 \mathrm{H}, \mathrm{CH}_{3} \mathrm{C}_{6} \mathrm{H}_{4} \mathrm{SO}_{3}{ }^{-}\right.$), $7.81\left(\mathrm{~d}, J=8.1 \mathrm{~Hz}, 2 \mathrm{H}, \mathrm{CH}_{3} \mathrm{C}_{6} \mathrm{H}_{4} \mathrm{SO}_{3}{ }^{-}\right) .-{ }^{13} \mathrm{C} \mathrm{NMR}$ $\left(62.9 \mathrm{MHz}, \mathrm{CDCl}_{3}\right): \delta=11.4\left(\mathrm{CH}_{3}\right), 12.9\left(2 \times \mathrm{CH}_{3}\right)$, $13.0\left(2 \times \mathrm{CH}_{3}\right), 13.9\left(\mathrm{CH}_{3}\right), 20.9\left(\mathrm{CH}_{2}\right), 21.3\left(\mathrm{CH}_{3}\right), 22.5$ $\left(\mathrm{CH}_{2}\right), 26.6\left(\mathrm{CH}_{2}\right), 27.3\left(\mathrm{CH}_{2}\right), 27.4\left(\mathrm{CH}_{2}\right), 31.3\left(\mathrm{CH}_{2}\right)$, $44.0\left(\mathrm{CH}_{2} \mathrm{~N}\right), 44.1\left(\mathrm{CH}_{2} \mathrm{~N}\right), 44.6\left(\mathrm{CH}_{2} \mathrm{~N}\right), 49.0\left(\mathrm{CH}_{2} \mathrm{~N}\right)$, $51.3\left(\mathrm{CH}_{2} \mathrm{~N}\right), 51.4\left(\mathrm{CH}_{2} \mathrm{~N}\right), 126.2(2 \times-\mathrm{CH}=), 128.2(2 \times$ $-\mathrm{CH}=), 138.4\left(\mathrm{C}-\mathrm{CH}_{3}\right), 144.7\left(\mathrm{C}-\mathrm{SO}_{3}\right), 163.8\left(\mathrm{CN}_{3}{ }^{+}\right)$. Anal. for $\mathrm{C}_{26} \mathrm{H}_{49} \mathrm{~N}_{3} \mathrm{O}_{3} \mathrm{~S}(483.8) \times \mathrm{H}_{2} \mathrm{O}$ : calcd. C 62.24, H 10.24, N 8.37; found C 62.65, H 9.86, N 8.58.

$N, N$-Diethyl- $N^{\prime}, N^{\prime}$-di-n-propyl- $N^{\prime \prime}-n$-hexyl- $N^{\prime \prime}-n$ butylguanidinium p-toluenesulfonate $\left(\boldsymbol{C}_{4}-\mathrm{gTos}\right)$

This compound was obtained from $12.17 \mathrm{~g}(0.0533 \mathrm{~mol})$ of $n$-butyl tosylate and $13.74 \mathrm{~g}(0.0485 \mathrm{~mol})$ of guanidine 3 in DMF $\left(100 \mathrm{~cm}^{3}\right)$. - Yield: $22.1 \mathrm{~g}(89 \%) .-n_{\mathrm{D}}^{20}=$ 1.5123. - IR (film): $v=1535\left(\mathrm{CN}_{3}{ }^{+}\right), 1202\left(\mathrm{SO}_{3}{ }^{-}\right), 677$ (Ar). $-{ }^{1} \mathrm{H}$ NMR $\left(250.13 \mathrm{MHz}, \mathrm{CDCl}_{3}\right): \delta=0.74-1.02(\mathrm{~m}$, $\left.12 \mathrm{H}, 2 \times \mathrm{C}_{2} \mathrm{H}_{4} \mathrm{CH}_{3}, \mathrm{C}_{5} \mathrm{H}_{10} \mathrm{CH}_{3}, \mathrm{C}_{3} \mathrm{H}_{7} \mathrm{CH}_{3}\right), 1.05-1.88$ $\left(\mathrm{m}, 22 \mathrm{H}, 8 \times \mathrm{CH}_{2}, 2 \times \mathrm{CH}_{2} \mathrm{CH}_{3}\right), 2.32\left(\mathrm{~s}, 3 \mathrm{H}, \mathrm{CH}_{3}-\right.$ $\left.\mathrm{C}_{6} \mathrm{H}_{4} \mathrm{SO}_{3}{ }^{-}\right), 2.87-3.56\left(\mathrm{~m}, 12 \mathrm{H}\right.$, all $\left.\mathrm{CH}_{2} \mathrm{~N}\right), 7.10(\mathrm{~d}, J=$ $\left.7.9 \mathrm{~Hz}, 2 \mathrm{H}, \mathrm{CH}_{3} \mathrm{C}_{6} \mathrm{H}_{4} \mathrm{SO}_{3}{ }^{-}\right), 7.81(\mathrm{~d}, J=8.1 \mathrm{~Hz}, 2 \mathrm{H}$, $\left.\mathrm{CH}_{3} \mathrm{C}_{6} \mathrm{H}_{4} \mathrm{SO}_{3}{ }^{-}\right) .{ }^{13} \mathrm{C} \mathrm{NMR}\left(62.9 \mathrm{MHz}, \mathrm{CDCl}_{3}\right): \delta=11.2$ $\left(\mathrm{CH}_{3}\right), 11.4\left(\mathrm{CH}_{3}\right), 12.9\left(2 \times \mathrm{CH}_{3}\right), 13.7\left(\mathrm{CH}_{3}\right), 13.9\left(\mathrm{CH}_{3}\right)$, $20.0\left(\mathrm{CH}_{2}\right), 20.9\left(\mathrm{CH}_{2}\right), 21.0\left(\mathrm{CH}_{2}\right), 21.3\left(\mathrm{CH}_{3}\right), 22.5$ $\left(\mathrm{CH}_{2}\right), 26.6\left(\mathrm{CH}_{2}\right), 27.4\left(\mathrm{CH}_{2}\right), 29.5\left(\mathrm{CH}_{2}\right), 31.3\left(\mathrm{CH}_{2}\right)$, $44.0\left(\mathrm{CH}_{2} \mathrm{~N}\right), 44.1\left(\mathrm{CH}_{2} \mathrm{~N}\right), 49.6\left(\mathrm{CH}_{2} \mathrm{~N}\right), 49.7\left(\mathrm{CH}_{2} \mathrm{~N}\right)$, $51.3\left(\mathrm{CH}_{2} \mathrm{~N}\right), 51.4\left(\mathrm{CH}_{2} \mathrm{~N}\right), 126.2(2 \times-\mathrm{CH}=), 128.2(2 \times$

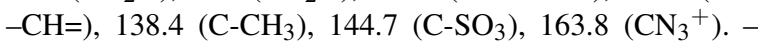
Anal. for $\mathrm{C}_{28} \mathrm{H}_{53} \mathrm{~N}_{3} \mathrm{O}_{3} \mathrm{~S}(511.8) \times \mathrm{H}_{2} \mathrm{O}$ : calcd. C 63.47, H 10.46, N 7.93; found C 63.55, H 10.11, N 8.39.

$N, N$-Diethyl- $N^{\prime}, N^{\prime}$-di-n-propyl- $N^{\prime \prime}, N^{\prime \prime}$-di-nhexylguanidinium p-toluenesulfonate $\left(\boldsymbol{C}_{6}-\mathbf{g T o s}\right)$

This compound was obtained from $12.09 \mathrm{~g}(0.0472 \mathrm{~mol})$ of $n$-hexyl tosylate and $12.15 \mathrm{~g}(0.0429 \mathrm{~mol})$ of guanidine
3 in DMF $\left(95 \mathrm{~cm}^{3}\right)$. - Yield: $20.36 \mathrm{~g}(88 \%) .-n_{\mathrm{D}}^{20}=$ 1.508. - IR (film): $v=1535\left(\mathrm{CN}_{3}{ }^{+}\right), 1202\left(\mathrm{SO}_{3}{ }^{-}\right), 677$ (Ar). $\left.-{ }^{1} \mathrm{H} \mathrm{NMR} \mathrm{(250.13} \mathrm{MHz,} \mathrm{CDCl}_{3}\right): \delta=0.74-1.01$ $\left(\mathrm{m}, 12 \mathrm{H}, 2 \times \mathrm{C}_{2} \mathrm{H}_{4} \mathrm{CH}_{3}, 2 \times \mathrm{C}_{5} \mathrm{H}_{10} \mathrm{CH}_{3}\right), 1.04-1.94$ $\left(\mathrm{m}, 26 \mathrm{H}, 10 \times \mathrm{CH}_{2}, 2 \times \mathrm{CH}_{2} \mathrm{CH}_{3}\right), 2.32\left(\mathrm{~s}, 3 \mathrm{H}, \mathrm{CH}_{3}-\right.$ $\left.\mathrm{C}_{6} \mathrm{H}_{4} \mathrm{SO}_{3}{ }^{-}\right), 2.90-3.57\left(\mathrm{~m}, 12 \mathrm{H}\right.$, all $\left.C_{2} \mathrm{~N}\right), 7.11(\mathrm{~d}, J=$ $\left.7.9 \mathrm{~Hz}, 2 \mathrm{H}, \mathrm{CH}_{3} C_{6} H_{4} \mathrm{SO}_{3}{ }^{-}\right), 7.82(\mathrm{~d}, J=8.1 \mathrm{~Hz}, 2 \mathrm{H}$, $\left.\mathrm{CH}_{3} \mathrm{C}_{6} \mathrm{H}_{4} \mathrm{SO}_{3}{ }^{-}\right) .{ }^{13} \mathrm{C} \mathrm{NMR}\left(62.9 \mathrm{MHz}, \mathrm{CDCl}_{3}\right): \delta=11.3$ $\left(\mathrm{CH}_{3}\right), 11.4\left(\mathrm{CH}_{3}\right), 12.9\left(\mathrm{CH}_{3}\right), 13.0\left(\mathrm{CH}_{3}\right), 13.9\left(\mathrm{CH}_{3}\right)$, $14.0\left(\mathrm{CH}_{3}\right), 20.9\left(\mathrm{CH}_{2}\right), 21.0\left(\mathrm{CH}_{2}\right), 21.3\left(\mathrm{CH}_{3}\right), 22.5(2 \times$ $\left.\mathrm{CH}_{2}\right), 26.5\left(\mathrm{CH}_{2}\right), 26.7\left(\mathrm{CH}_{2}\right), 27.4\left(\mathrm{CH}_{2}\right), 27.5\left(\mathrm{CH}_{2}\right), 31.3$ $\left(\mathrm{CH}_{2}\right), 31.4\left(\mathrm{CH}_{2}\right), 44.0\left(\mathrm{CH}_{2} \mathrm{~N}\right), 44.2\left(\mathrm{CH}_{2} \mathrm{~N}\right), 49.6(2 \times$ $\left.\mathrm{CH}_{2} \mathrm{~N}\right), 51.3\left(\mathrm{CH}_{2} \mathrm{~N}\right), 51.5\left(\mathrm{CH}_{2} \mathrm{~N}\right), 126.2(2 \times-\mathrm{CH}=)$, $128.3(2 \times-\mathrm{CH}=), 138.4\left(\mathrm{C}-\mathrm{CH}_{3}\right), 144.5\left(\mathrm{C}-\mathrm{SO}_{3}\right), 164.0$ $\left(\mathrm{CN}_{3}{ }^{+}\right)$. - Anal. for $\mathrm{C}_{30} \mathrm{H}_{57} \mathrm{~N}_{3} \mathrm{O}_{3} \mathrm{~S}(539.9) \times \mathrm{H}_{2} \mathrm{O}$ : calcd. C 64.59, H 10.66, N 7.53; found C 64.38, H 10.11, N 7.93.

$N, N$-Diethyl- $N^{\prime}, N^{\prime}$-di-n-propyl- $N^{\prime \prime}-n$-hexyl- $N^{\prime \prime}-n$ octylguanidinium p-toluenesulfonate $\left(\boldsymbol{C}_{\mathbf{8}}-\mathbf{g T o s}\right)$

This compound was obtained from $9.78 \mathrm{~g}(0.0344 \mathrm{~mol})$ of $n$-octyl tosylate and $8.86 \mathrm{~g}(0.0313 \mathrm{~mol})$ of guanidine 3 in DMF $\left(80 \mathrm{~cm}^{3}\right)$. - Yield: $16.20 \mathrm{~g}(83 \%) .-n_{\mathrm{D}}^{20}=$ 1.5034. - IR (film): $v=1534\left(\mathrm{CN}_{3}^{+}\right), 1202\left(\mathrm{SO}_{3}{ }^{-}\right), 677$ (Ar). $-{ }^{1} \mathrm{H}$ NMR (250.13 MHz, $\left.\mathrm{CDCl}_{3}\right): \delta=0.77-1.00$ $\left(\mathrm{m}, 12 \mathrm{H}, 2 \times \mathrm{C}_{2} \mathrm{H}_{4} \mathrm{CH}_{3}, \mathrm{C}_{7} \mathrm{H}_{14} \mathrm{CH}_{3}, \mathrm{C}_{5} \mathrm{H}_{10} \mathrm{CH}_{3}\right), 1.04-$ $1.91\left(\mathrm{~m}, 30 \mathrm{H}, 12 \times \mathrm{CH}_{2}, 2 \times \mathrm{CH}_{2} \mathrm{CH}_{3}\right), 2.32(\mathrm{~s}, 3 \mathrm{H}$, $\left.\mathrm{CH}_{3}-\mathrm{C}_{6} \mathrm{H}_{4} \mathrm{SO}_{3}{ }^{-}\right), 2.85-3.51\left(\mathrm{~m}, 12 \mathrm{H}\right.$, all $\left.\mathrm{CH}_{2} \mathrm{~N}\right), 7.10$ $\left(\mathrm{d}, J=7.9 \mathrm{~Hz}, 2 \mathrm{H}, \mathrm{CH}_{3} \mathrm{C}_{6} \mathrm{H}_{4} \mathrm{SO}_{3}{ }^{-}\right), 7.82(\mathrm{~d}, J=8.1 \mathrm{~Hz}$, $\left.2 \mathrm{H}, \mathrm{CH}_{3} \mathrm{C}_{6} \mathrm{H}_{4} \mathrm{SO}_{3}{ }^{-}\right) .-{ }^{13} \mathrm{C} \mathrm{NMR}\left(62.9 \mathrm{MHz}, \mathrm{CDCl}_{3}\right)$ : $\delta=11.3\left(\mathrm{CH}_{3}\right), 11.4\left(\mathrm{CH}_{3}\right), 12.9\left(\mathrm{CH}_{3}\right), 13.0\left(\mathrm{CH}_{3}\right), 13.9$ $\left(\mathrm{CH}_{3}\right), 14.1\left(\mathrm{CH}_{3}\right), 20.9\left(\mathrm{CH}_{2}\right), 21.0\left(\mathrm{CH}_{2}\right), 21.2\left(\mathrm{CH}_{3}\right)$, $22.5\left(\mathrm{CH}_{2}\right), 22.6\left(\mathrm{CH}_{2}\right), 26.5\left(\mathrm{CH}_{2}\right), 26.6\left(\mathrm{CH}_{2}\right), 26.8$ $\left(\mathrm{CH}_{2}\right), 26.9\left(\mathrm{CH}_{2}\right), 27.4\left(\mathrm{CH}_{2}\right), 29.1\left(\mathrm{CH}_{2}\right), 31.3\left(\mathrm{CH}_{2}\right)$, $31.7\left(\mathrm{CH}_{2}\right), 44.0\left(\mathrm{CH}_{2} \mathrm{~N}\right), 44.1\left(\mathrm{CH}_{2} \mathrm{~N}\right), 49.7\left(2 \times \mathrm{CH}_{2} \mathrm{~N}\right)$, $51.4\left(\mathrm{CH}_{2} \mathrm{~N}\right), 51.5\left(\mathrm{CH}_{2} \mathrm{~N}\right), 126.2(2 \times-\mathrm{CH}=), 128.2(2 \times$

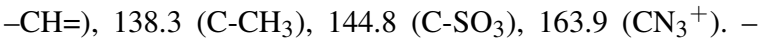
Anal. for $\mathrm{C}_{32} \mathrm{H}_{61} \mathrm{~N}_{3} \mathrm{O}_{3} \mathrm{~S}(567.9) \times 0.5 \mathrm{H}_{2} \mathrm{O}$ : calcd. C 66.62, H 10.83, N 7.28; found C 66.10, H 10.49, N 7.74.

$N, N$-Diethyl- $N^{\prime}, N^{\prime}$-di-n-propyl- $N^{\prime \prime}-n$-hexyl- $N^{\prime \prime}-n$ decylguanidinium p-toluenesulfonate $\left(\boldsymbol{C}_{10}-\mathrm{gTos}\right)$

This compound was obtained from $12.62 \mathrm{~g}(0.0468 \mathrm{~mol})$ of $n$-decyl tosylate and $12.06 \mathrm{~g}(0.0425 \mathrm{~mol})$ of guanidine 3 in DMF $\left(100 \mathrm{~cm}^{3}\right)$. - Yield: $23.58 \mathrm{~g}(93 \%) .-n_{\mathrm{D}}^{20}=$ 1.5007. - IR (film): $v=1535\left(\mathrm{CN}_{3}{ }^{+}\right), 1202\left(\mathrm{SO}_{3}{ }^{-}\right), 678$ (Ar). $-{ }^{1} \mathrm{H}$ NMR (250.13 MHz, $\left.\mathrm{CDCl}_{3}\right): \delta=0.77-1.00$ $\left(\mathrm{m}, 12 \mathrm{H}, 2 \times \mathrm{C}_{2} \mathrm{H}_{4} \mathrm{CH}_{3}, \mathrm{C}_{9} \mathrm{H}_{18} \mathrm{CH}_{3}, \mathrm{C}_{5} \mathrm{H}_{10} \mathrm{CH}_{3}\right), 1.07-$ $1.92\left(\mathrm{~m}, 34 \mathrm{H}, 14 \times \mathrm{CH}_{2}, 2 \times \mathrm{CH}_{2} \mathrm{CH}_{3}\right), 2.31(\mathrm{~s}, 3 \mathrm{H}$, $\left.\mathrm{CH}_{3}-\mathrm{C}_{6} \mathrm{H}_{4} \mathrm{SO}_{3}{ }^{-}\right), 2.88-3.52\left(\mathrm{~m}, 12 \mathrm{H}\right.$, all $\left.\mathrm{CH}_{2} \mathrm{~N}\right), 7.09$ (d, $\left.J=7.1 \mathrm{~Hz}, 2 \mathrm{H}, \mathrm{CH}_{3} C_{6} H_{4} \mathrm{SO}_{3}{ }^{-}\right), 7.82(\mathrm{~d}, J=8.1 \mathrm{~Hz}$, $\left.2 \mathrm{H}, \mathrm{CH}_{3} \mathrm{C}_{6} \mathrm{H}_{4} \mathrm{SO}_{3}{ }^{-}\right) .-{ }^{13} \mathrm{C} \mathrm{NMR}\left(62.9 \mathrm{MHz}, \mathrm{CDCl}_{3}\right)$ : $\delta=11.3\left(\mathrm{CH}_{3}\right), 11.4\left(\mathrm{CH}_{3}\right), 12.9\left(\mathrm{CH}_{3}\right), 13.0\left(\mathrm{CH}_{3}\right), 13.9$ $\left(\mathrm{CH}_{3}\right), 14.1\left(\mathrm{CH}_{3}\right), 20.9\left(\mathrm{CH}_{2}\right), 21.0\left(\mathrm{CH}_{2}\right), 21.3\left(\mathrm{CH}_{3}\right)$, 
$22.5\left(\mathrm{CH}_{2}\right), 22.6\left(\mathrm{CH}_{2}\right), 26.5\left(\mathrm{CH}_{2}\right), 26.6\left(\mathrm{CH}_{2}\right), 26.8$ $\left(\mathrm{CH}_{2}\right), 26.9\left(\mathrm{CH}_{2}\right), 27.4\left(\mathrm{CH}_{2}\right), 27.5\left(\mathrm{CH}_{2}\right), 29.2\left(\mathrm{CH}_{2}\right)$, $29.5\left(\mathrm{CH}_{2}\right), 31.3\left(\mathrm{CH}_{2}\right), 31.4\left(\mathrm{CH}_{2}\right), 44.0\left(\mathrm{CH}_{2} \mathrm{~N}\right), 44.1$ $\left(\mathrm{CH}_{2} \mathrm{~N}\right), 50.0\left(2 \times \mathrm{CH}_{2} \mathrm{~N}\right), 51.4\left(\mathrm{CH}_{2} \mathrm{~N}\right), 51.5\left(\mathrm{CH}_{2} \mathrm{~N}\right)$, $126.2(2 \times-\mathrm{CH}=), 128.2(2 \times-\mathrm{CH}=), 138.1\left(\mathrm{C}-\mathrm{CH}_{3}\right), 145.0$ $\left(\mathrm{C}-\mathrm{SO}_{3}\right), 163.9\left(\mathrm{CN}_{3}{ }^{+}\right)$. - Anal. for $\mathrm{C}_{34} \mathrm{H}_{65} \mathrm{~N}_{3} \mathrm{O}_{3} \mathrm{~S}(596.0)$ $\times 0.5 \mathrm{H}_{2} \mathrm{O}$ : calcd. C 67.50, H 11.00, N 6.95; found C 67.51, H 10.87, N 6.82 .

General procedure for anion metathesis reactions. Synthesis of guanidinium tetrafluoroborates $\boldsymbol{C}_{\boldsymbol{n}}-\mathbf{g} \boldsymbol{B} \boldsymbol{F}_{\mathbf{4}}$, acesulfamates $\boldsymbol{C}_{\boldsymbol{n}}$-gAce, and saccharinates $\boldsymbol{C}_{\boldsymbol{n}} \mathbf{- g S a c}(n=3,4,6,8,10)$

Metathesis reaction in methanol

A methanol solution of the RTIL $\mathbf{C}_{\boldsymbol{n}}$-gCl was slowly added dropwise to a stirred suspension of sodium tetrafluoroborate in methanol at $60{ }^{\circ} \mathrm{C}$. The flask was equipped with a magnetic stirrer, a dropping funnel, a reflux condenser and a calcium chloride tube. The reaction mixture was stirred for $4 \mathrm{~h}$ at reflux and cooled to r.t., and the formed white precipitate of sodium chloride was removed by filtration. The filtrate was distilled under reduced pressure giving the crude product of the metathesis reaction. In order to remove the residual sodium chloride, the residue was dissolved in a small quantity of dry THF, filtered, and the solvent was evaporated on a rotary evaporator. The residue was further dissolved in a small quantity of dichloromethane and washed with distilled water until the probe for chloride anions $\left(\mathrm{AgNO}_{3}\right.$ test $)$ was negative. The dichloromethane layer was dried with sodium sulfate and filtered, and dichloromethane was evaporated on a rotary evaporator. The resulting slight yellow RTILs $\mathbf{C}_{\boldsymbol{n}}-\mathbf{g B} \mathbf{g B}_{\mathbf{4}}$ were further dried in vacuo $\left(90{ }^{\circ} \mathrm{C} / 0.01 \mathrm{mbar}\right)$ for $6-8 \mathrm{~h}$.

\section{Metathesis reaction in water}

A solution of the RTIL $\mathbf{C}_{\boldsymbol{n}}$-gCl in water was slowly added dropwise to a saturated stirred solution of the corresponding sodium or potassium salt in water at $60{ }^{\circ} \mathrm{C}$. The flask was equipped with a magnetic stirrer, a dropping funnel, a reflux condenser, and a calcium chloride tube. The reaction mixture was stirred for $4 \mathrm{~h}$ at $60{ }^{\circ} \mathrm{C}$ and then left to cool over night. The resulting two layers were separated, and the product of the metathesis reaction (the top layer) was dissolved in dichloromethane and washed with water until the probe for chloride anions $\left(\mathrm{AgNO}_{3}\right.$ test $)$ was negative. The dichloromethane layer was dried with sodium sulfate and filtered, and dichloromethane was evaporated on a rotary evaporator. The resulting slightly yellow RTILs $\mathbf{C}_{\boldsymbol{n}}$-gX were further dried in vacuo $\left(90{ }^{\circ} \mathrm{C} / 0.01 \mathrm{mbar}\right)$ for $6-8 \mathrm{~h}$.

$N, N$-Diethyl- $N^{\prime}, N^{\prime}$-di-n-propyl- $N^{\prime \prime}-n$-hexyl- $N^{\prime \prime}-n$ propylguanidinium tetrafluoroborate $\left(\boldsymbol{C}_{\mathbf{3}}-\mathbf{g} \boldsymbol{B F}_{\mathbf{4}}\right)$

This compound was obtained from $7.02 \mathrm{~g}(0.019 \mathrm{~mol})$ of $\mathbf{C}_{3}-\mathbf{g C l}$ in $\mathrm{CH}_{3} \mathrm{OH}\left(45 \mathrm{~cm}^{3}\right)$ and $2.52 \mathrm{~g}(0.023 \mathrm{~mol})$ of
$\mathrm{NaBF}_{4}$ in $\mathrm{CH}_{3} \mathrm{OH}\left(25 \mathrm{~cm}^{3}\right)$. - Yield $7.7 \mathrm{~g}(98 \%) .-n_{\mathrm{D}}^{20}=$ 1.4578. - IR (film): $v=1534\left(\mathrm{CN}_{3}{ }^{+}\right), 1034$ and 1047 (BF) $\mathrm{cm}^{-1} .-{ }^{1} \mathrm{H}$ NMR $\left(250.13 \mathrm{MHz}, \mathrm{CDCl}_{3}\right): \delta=0.83-$ $1.01\left(\mathrm{~m}, 12 \mathrm{H}, 3 \times \mathrm{C}_{2} \mathrm{H}_{4} \mathrm{CH}_{3}, \mathrm{C}_{5} \mathrm{H}_{10} \mathrm{CH}_{3}\right), 1.15-1.89(\mathrm{~m}$, $\left.20 \mathrm{H}, 7 \times \mathrm{CH}_{2}, 2 \times \mathrm{CH}_{2} \mathrm{CH}_{3}\right), 2.93-3.49(\mathrm{~m}, 12 \mathrm{H}$, all $\left.\mathrm{CH}_{2} \mathrm{~N}\right) .-{ }^{13} \mathrm{C}$ NMR $\left(62.9 \mathrm{MHz}, \mathrm{CDCl}_{3}\right): \delta=11.2(2 \times$ $\left.\mathrm{CH}_{3}\right), 11.3\left(\mathrm{CH}_{3}\right), 12.7\left(\mathrm{CH}_{3}\right), 13.9\left(2 \times \mathrm{CH}_{3}\right), 20.8\left(\mathrm{CH}_{2}\right)$, $22.5\left(3 \times \mathrm{CH}_{2}\right), 26.5\left(\mathrm{CH}_{2}\right), 27.4\left(\mathrm{CH}_{2}\right), 31.3\left(\mathrm{CH}_{2}\right), 43.8$ $\left(2 \times \mathrm{CH}_{2} \mathrm{~N}\right), 49.6\left(\mathrm{CH}_{2} \mathrm{~N}\right), 51.3\left(3 \times \mathrm{CH}_{2} \mathrm{~N}\right), 164.1(\mathrm{~s}$, $\mathrm{CN}_{3}{ }^{+}$). - Anal. for $\mathrm{C}_{20} \mathrm{H}_{44} \mathrm{BF}_{4} \mathrm{~N}_{3}$ (413.4): calcd. C 58.11, H 10.73, N 10.16; found C 57.52, H 10.49, N 10.29.

$N, N$-Diethyl- $N^{\prime}, N^{\prime}$-di-n-propyl- $N^{\prime \prime}-n$-hexyl- $N^{\prime \prime}-n$ butylguanidinium tetrafluoroborate $\left(\boldsymbol{C}_{\mathbf{4}}-\boldsymbol{g} \boldsymbol{B F}_{4}\right)$

This compound was obtained from $9.16 \mathrm{~g}(0.025 \mathrm{~mol})$ of $\mathbf{C}_{4}-\mathbf{g C l}$ in $\mathrm{CH}_{3} \mathrm{OH}\left(60 \mathrm{~cm}^{3}\right)$ and $3.18 \mathrm{~g}(0.03 \mathrm{~mol})$ of $\mathrm{NaBF}_{4}$ in $\mathrm{CH}_{3} \mathrm{OH}\left(30 \mathrm{~cm}^{3}\right)$. - Yield $10.2 \mathrm{~g}(96 \%) .-n_{\mathrm{D}}^{20}=$ 1.4572. - IR (film): $v=1534\left(\mathrm{CN}_{3}{ }^{+}\right), 1033$ and 1048 (BF) $\mathrm{cm}^{-1} .{ }^{1} \mathrm{H}$ NMR $\left(250.13 \mathrm{MHz}, \mathrm{CDCl}_{3}\right): \delta=0.81-1.02$ $\left(\mathrm{m}, 12 \mathrm{H}, 2 \times \mathrm{C}_{2} \mathrm{H}_{4} \mathrm{CH}_{3}, \mathrm{C}_{3} \mathrm{H}_{6} \mathrm{CH}_{3}, \mathrm{C}_{5} \mathrm{H}_{10} \mathrm{CH}_{3}\right), 1.14-$ $1.90\left(\mathrm{~m}, 22 \mathrm{H}, 8 \times \mathrm{CH}_{2}, 2 \times \mathrm{CH}_{2} \mathrm{CH}_{3}\right), 2.92-3.51(\mathrm{~m}$, $12 \mathrm{H}$, all $\left.\mathrm{CH}_{2} \mathrm{~N}\right) .-{ }^{13} \mathrm{C} \mathrm{NMR}\left(62.9 \mathrm{MHz}, \mathrm{CDCl}_{3}\right): \delta=11.3$ $\left(2 \times \mathrm{CH}_{3}\right), 12.8\left(\mathrm{CH}_{3}\right), 12.9\left(\mathrm{CH}_{3}\right), 13.9\left(2 \times \mathrm{CH}_{3}\right), 20.1$ $\left(\mathrm{CH}_{2}\right), 20.8\left(\mathrm{CH}_{2}\right), 22.5\left(2 \times \mathrm{CH}_{2}\right), 26.6\left(\mathrm{CH}_{2}\right), 27.4\left(\mathrm{CH}_{2}\right)$, $29.5\left(\mathrm{CH}_{2}\right), 31.3\left(\mathrm{CH}_{2}\right), 43.8\left(2 \times \mathrm{CH}_{2} \mathrm{~N}\right), 49.4\left(\mathrm{CH}_{2} \mathrm{~N}\right)$, $49.6\left(\mathrm{CH}_{2} \mathrm{~N}\right), 51.4\left(2 \times \mathrm{CH}_{2} \mathrm{~N}\right), 164.0\left(\mathrm{CN}_{3}{ }^{+}\right),-$Anal. for $\mathrm{C}_{21} \mathrm{H}_{46} \mathrm{BF}_{4} \mathrm{~N}_{3}(427.4) \times \mathrm{H}_{2} \mathrm{O}$ : calcd. C 57.79, $\mathrm{H} 10.85$, N 9.63; found C 58.19, H 10.62, N 10.07.

\section{$N, N$-Diethyl- $N^{\prime}, N^{\prime}$-di-n-propyl- $N^{\prime \prime}, N^{\prime \prime}-$ di-n- hexylguanidinium tetrafluoroborate $\left(\boldsymbol{C}_{6}-\mathbf{g} \boldsymbol{B F}_{4}\right)$}

This compound was obtained from $12.59 \mathrm{~g}(0.031 \mathrm{~mol})$ of $\mathbf{C}_{\mathbf{6}}-\mathbf{g C l}$ in $\mathrm{CH}_{3} \mathrm{OH}\left(80 \mathrm{~cm}^{3}\right)$ and $4.1 \mathrm{~g}(0.037 \mathrm{~mol})$ of $\mathrm{NaBF}_{4}$ in $\mathrm{CH}_{3} \mathrm{OH}\left(40 \mathrm{~cm}^{3}\right)$. - Yield $13.4 \mathrm{~g}(94 \%) .-n_{\mathrm{D}}^{20}=$ 1.4597. - IR (film): $v=1533\left(\mathrm{CN}_{3}{ }^{+}\right), 1033$ and 1047 (BF) $\mathrm{cm}^{-1} .-{ }^{1} \mathrm{H}$ NMR $\left(250.13 \mathrm{MHz}, \mathrm{CDCl}_{3}\right): \delta=0.81-$ $1.01\left(\mathrm{~m}, 12 \mathrm{H}, 2 \times \mathrm{C}_{2} \mathrm{H}_{4} \mathrm{CH}_{3}, 2 \times \mathrm{C}_{5} \mathrm{H}_{10} \mathrm{CH}_{3}\right), 1.15-$ $1.89\left(\mathrm{~m}, 26 \mathrm{H}, 10 \times \mathrm{CH}_{2}, 2 \times \mathrm{CH}_{2} \mathrm{CH}_{3}\right), 2.91-3.53(\mathrm{~m}$, $12 \mathrm{H}$, all $\left.\mathrm{CH}_{2} \mathrm{~N}\right) .-{ }^{13} \mathrm{C} \mathrm{NMR}\left(62.9 \mathrm{MHz}, \mathrm{CDCl}_{3}\right): \delta=$ $11.2\left(\mathrm{CH}_{3}\right), 11.3\left(\mathrm{CH}_{3}\right), 12.7\left(\mathrm{CH}_{3}\right), 12.8\left(\mathrm{CH}_{3}\right), 13.9(2 \times$ $\left.\mathrm{CH}_{3}\right), 20.8\left(\mathrm{CH}_{2}\right), 20.9\left(\mathrm{CH}_{2}\right), 22.5\left(2 \times \mathrm{CH}_{2}\right), 26.5\left(\mathrm{CH}_{2}\right)$, $26.6\left(\mathrm{CH}_{2}\right), 27.3\left(\mathrm{CH}_{2}\right), 27.4\left(\mathrm{CH}_{2}\right), 31.3\left(2 \times \mathrm{CH}_{2}\right), 43.8$ $\left(\mathrm{CH}_{2} \mathrm{~N}\right), 43.9\left(\mathrm{CH}_{2} \mathrm{~N}\right), 49.7\left(2 \times \mathrm{CH}_{2} \mathrm{~N}\right), 51.3\left(\mathrm{CH}_{2} \mathrm{~N}\right), 51.4$ $\left(\mathrm{CH}_{2} \mathrm{~N}\right), 164.0\left(\mathrm{CN}_{3}{ }^{+}\right)$. - Anal. for $\mathrm{C}_{23} \mathrm{H}_{50} \mathrm{BF}_{4} \mathrm{~N}_{3}(455.5)$ : calcd. C 60.65, H 11.06, N 9.23; found C 60.48, H 10.91, N 9.16.

$N, N$-Diethyl- $N^{\prime}, N^{\prime}$-di-n-propyl- $N^{\prime \prime}-n$-hexyl- $N^{\prime \prime}-n$ octylguanidinium tetrafluoroborate $\left(\boldsymbol{C}_{\mathbf{8}}-\mathbf{g} \boldsymbol{B F}_{\mathbf{4}}\right)$

This compound was obtained from $17.9 \mathrm{~g}(0.041 \mathrm{~mol})$ of $\mathbf{C}_{\mathbf{8}}$ - $\mathbf{g C l}$ in $\mathrm{CH}_{3} \mathrm{OH}\left(110 \mathrm{~cm}^{3}\right)$ and $5.45 \mathrm{~g}(0.049 \mathrm{~mol})$ 
$\mathrm{NaBF}_{4}$ in $\mathrm{CH}_{3} \mathrm{OH}\left(55 \mathrm{~cm}^{3}\right)$. - Yield $=19.6 \mathrm{~g}(98 \%)$. $n_{\mathrm{D}}^{20}=1.4580 .-\mathrm{IR}$ (film): $v=1534\left(\mathrm{CN}_{3}{ }^{+}\right), 1034$ and 1048 (B-F) $\mathrm{cm}^{-1} .{ }^{1} \mathrm{H}$ NMR $\left(250.13 \mathrm{MHz}, \mathrm{CDCl}_{3}\right): \delta=0.79-$ $0.99\left(\mathrm{~m}, 12 \mathrm{H}, 2 \times \mathrm{C}_{2} \mathrm{H}_{4} \mathrm{CH}_{3}, \mathrm{C}_{5} \mathrm{H}_{10} \mathrm{CH}_{3}, \mathrm{C}_{7} \mathrm{H}_{14} \mathrm{CH}_{3}\right)$, $1.17-1.84\left(\mathrm{~m}, 30 \mathrm{H}, \mathrm{I} \times \mathrm{CH}_{2}, 2 \times \mathrm{CH}_{2} \mathrm{CH}_{3}\right), 2.93-3.52$ $\left(\mathrm{m}, 12 \mathrm{H}\right.$, all $\left.\mathrm{CH}_{2} \mathrm{~N}\right) .-{ }^{13} \mathrm{C} \mathrm{NMR}\left(62.9 \mathrm{MHz}, \mathrm{CDCl}_{3}\right)$ : $\delta=11.3\left(2 \times \mathrm{CH}_{3}\right), 12.7\left(\mathrm{CH}_{3}\right), 12.8\left(\mathrm{CH}_{3}\right), 14.0(2 \times$ $\left.\mathrm{CH}_{3}\right), 20.8\left(\mathrm{CH}_{2}\right), 20.9\left(\mathrm{CH}_{2}\right), 22.5\left(\mathrm{CH}_{2}\right), 22.6\left(\mathrm{CH}_{2}\right), 26.5$ $\left(\mathrm{CH}_{2}\right), 27.4\left(\mathrm{CH}_{2}\right), 28.9\left(\mathrm{CH}_{2}\right), 29.2\left(\mathrm{CH}_{2}\right), 30.3\left(2 \times \mathrm{CH}_{2}\right)$, $31.3\left(\mathrm{CH}_{2}\right), 31.7\left(\mathrm{CH}_{2}\right), 43.8\left(2 \times \mathrm{CH}_{2} \mathrm{~N}\right), 43.9\left(\mathrm{CH}_{2} \mathrm{~N}\right)$, $49.6\left(\mathrm{CH}_{2} \mathrm{~N}\right), 51.4\left(2 \times \mathrm{CH}_{2} \mathrm{~N}\right), 164.0\left(\mathrm{CN}_{3}{ }^{+}\right)$. - Anal. for $\mathrm{C}_{25} \mathrm{H}_{54} \mathrm{BF}_{4} \mathrm{~N}_{3}(483.5) \times 0.5 \mathrm{H}_{2} \mathrm{O}$ : calcd. C 60.96, H 11.26, N 8.53; found C $61.00, \mathrm{H} 11.11$, N 9.11.

\section{$N, N$-Diethyl- $N^{\prime}, N^{\prime}$-di-n-propyl- $N^{\prime \prime}-n$-hexyl- $N^{\prime \prime}-n$ - decylguanidinium tetrafluoroborate $\left(\boldsymbol{C}_{\mathbf{1 0}}-\mathbf{g} \boldsymbol{B F}_{\mathbf{4}}\right)$}

This compound was obtained from $18.00 \mathrm{~g}(0.039 \mathrm{~mol})$ of $\mathbf{C}_{10}$-gCl in $\mathrm{CH}_{3} \mathrm{OH}\left(115 \mathrm{~cm}^{3}\right)$ and $5.15 \mathrm{~g}(0.047 \mathrm{~mol})$ $\mathrm{NaBF}_{4}$ in $\mathrm{CH}_{3} \mathrm{OH}\left(52 \mathrm{~cm}^{3}\right)$. - Yield $=19.4 \mathrm{~g}(97 \%)$. $n_{\mathrm{D}}^{20}=1.4567 .-\mathrm{IR}($ film $): v=1535\left(\mathrm{CN}_{3}{ }^{+}\right), 1034$ and 1048 (B-F) $\mathrm{cm}^{-1} .{ }^{1}{ }^{1} \mathrm{H}$ NMR $\left(250.13 \mathrm{MHz}, \mathrm{CDCl}_{3}\right): \delta=0.79-$ $1.01\left(\mathrm{~m}, 12 \mathrm{H}, 2 \times \mathrm{C}_{2} \mathrm{H}_{4} \mathrm{CH}_{3}, \mathrm{C}_{5} \mathrm{H}_{10} \mathrm{CH}_{3}, \mathrm{C}_{9} \mathrm{H}_{18} \mathrm{CH}_{3}\right)$, $1.13-1.91\left(\mathrm{~m}, 34 \mathrm{H}, \mathrm{I} \times \mathrm{CH}_{2}, 2 \times \mathrm{CH}_{2} \mathrm{CH}_{3}\right), 2.94-3.51$ $\left(\mathrm{m}, 12 \mathrm{H}\right.$, all $\left.\mathrm{CH}_{2} \mathrm{~N}\right),-{ }^{13} \mathrm{C}$ NMR $\left(62.9 \mathrm{MHz}, \mathrm{CDCl}_{3}\right)$ : $\delta=11.2\left(\mathrm{CH}_{3}\right), 11.3\left(\mathrm{CH}_{3}\right), 12.7\left(\mathrm{CH}_{3}\right), 12.8\left(\mathrm{CH}_{3}\right), 14.0$ $\left(\mathrm{CH}_{3}\right), 14.1\left(\mathrm{CH}_{3}\right), 20.8\left(\mathrm{CH}_{2}\right), 20.9\left(\mathrm{CH}_{2}\right), 22.5\left(\mathrm{CH}_{2}\right)$, $22.6\left(\mathrm{CH}_{2}\right), 26.5\left(\mathrm{CH}_{2}\right), 27.3\left(\mathrm{CH}_{2}\right), 27.4\left(2 \times \mathrm{CH}_{2}\right), 29.2$ $\left(2 \times \mathrm{CH}_{2}\right), 29.5\left(2 \times \mathrm{CH}_{2}\right), 31.3\left(\mathrm{CH}_{2}\right), 31.8\left(\mathrm{CH}_{2}\right), 43.9$ $\left(2 \times \mathrm{CH}_{2} \mathrm{~N}\right), 49.6\left(\mathrm{CH}_{2} \mathrm{~N}\right), 49.7\left(\mathrm{CH}_{2} \mathrm{~N}\right), 51.3\left(2 \times \mathrm{CH}_{2} \mathrm{~N}\right)$, $164.0\left(\mathrm{CN}_{3}{ }^{+}\right)$. - Anal. for $\mathrm{C}_{27} \mathrm{H}_{58} \mathrm{BF}_{4} \mathrm{~N}_{3}(511.6) \times 0.5$ $\mathrm{H}_{2} \mathrm{O}$ : calcd. C 62.29, H 11.42, N 8.07; found C 62.00, H 11.33, N, 8.67.

\section{$N, N$-Diethyl- $N^{\prime}, N^{\prime}$-di-n-propyl- $N^{\prime \prime}-n$-hexyl- $N^{\prime \prime}-n$ - propylguanidinium acesulfamate $\left(\boldsymbol{C}_{\mathbf{3}}-\mathbf{g A c e}\right)$}

This compound was obtained from $7.18 \mathrm{~g}(0.021 \mathrm{~mol})$ of $\mathbf{C}_{3}-\mathbf{g C l}$ in $\mathrm{CH}_{3} \mathrm{OH}\left(4.14 \mathrm{~cm}^{3}\right)$ and $8.25 \mathrm{~g}(0.041 \mathrm{~mol})$ acesulfame- $\mathrm{K}$ in $\mathrm{CH}_{3} \mathrm{OH}\left(8 \mathrm{~cm}^{3}\right)$. - Yield $=9.2 \mathrm{~g}(92 \%)$. $n_{\mathrm{D}}^{20}=1.5032 .-\mathrm{IR}(\mathrm{film}): v=1533\left(\mathrm{CN}_{3}{ }^{+}\right), 1171\left(\mathrm{SO}_{2}\right)$, $1602(\mathrm{C}=\mathrm{C}), 1650(\mathrm{C}=\mathrm{O}) \mathrm{cm}^{-1} .-{ }^{1} \mathrm{H}$ NMR $(250.13 \mathrm{MHz}$, $\left.\mathrm{CDCl}_{3}\right): \delta=0.80-1.01\left(\mathrm{~m}, 12 \mathrm{H}, 3 \times \mathrm{C}_{2} \mathrm{H}_{4} \mathrm{CH}_{3}\right.$, $\left.\mathrm{C}_{5} \mathrm{H}_{10} \mathrm{CH}_{3}\right), 1.17-1.91\left(\mathrm{~m}, 20 \mathrm{H}, 7 \times \mathrm{CH}_{2}, 2 \times \mathrm{CH}_{2} \mathrm{CH}_{3}\right)$, $2.00\left(\mathrm{~d}, J=1.0 \mathrm{~Hz}, 3 \mathrm{H}, \mathrm{CH}_{3} /\right.$ Ace $), 2.95-3.50(\mathrm{~m}, 12 \mathrm{H}$, all $\left.\mathrm{CH}_{2} \mathrm{~N}\right), 5.46(\mathrm{~d}, J=1.0 \mathrm{~Hz}, 1 \mathrm{H}, \mathrm{CH} /$ Ace $) .-{ }^{13} \mathrm{C} \mathrm{NMR}$ $\left(62.9 \mathrm{MHz}, \mathrm{CDCl}_{3}\right): \delta=11.3\left(\mathrm{CH}_{3}\right), 11.4\left(\mathrm{CH}_{3}\right), 12.8$ $\left(\mathrm{CH}_{3}\right), 12.9\left(\mathrm{CH}_{3}\right), 13.9\left(2 \times \mathrm{CH}_{3}\right), 19.9\left(\mathrm{CH}_{3} /\right.$ Ace $), 20.9$ $\left(\mathrm{CH}_{2}\right), 22.5\left(3 \times \mathrm{CH}_{2}\right), 26.6\left(\mathrm{CH}_{2}\right), 27.5\left(\mathrm{CH}_{2}\right), 31.3\left(\mathrm{CH}_{2}\right)$, $44.0\left(\mathrm{CH}_{2} \mathrm{~N}\right), 44.1\left(\mathrm{CH}_{2} \mathrm{~N}\right), 49.7\left(\mathrm{CH}_{2} \mathrm{~N}\right), 51.4\left(3 \times \mathrm{CH}_{2} \mathrm{~N}\right)$, 102.4 (CH/Ace), 160.3 (C-O/Ace), $163.9\left(\mathrm{CN}_{3}{ }^{+}\right), 169.8$ $(\mathrm{C}=\mathrm{O} /$ Ace $)$. - Anal. for $\mathrm{C}_{24} \mathrm{H}_{48} \mathrm{~N}_{4} \mathrm{O}_{4} \mathrm{~S}(488.7) \times \mathrm{H}_{2} \mathrm{O}$ : calcd. C 56.88, H 9.95, N 11.06; found C 57.70, H 9.89, N 11.39.
$N, N$-Diethyl- $N^{\prime}, N^{\prime}$-di-n-propyl- $N^{\prime \prime}-n$-hexyl- $N^{\prime \prime}-n-$ butylguanidinium acesulfamate $\left(\boldsymbol{C}_{\mathbf{4}}-\mathbf{g A c e}\right)$

This compound was obtained from $20.58 \mathrm{~g}(0.056 \mathrm{~mol})$ of $\mathbf{C}_{4}-\mathbf{g C l}$ in $\mathrm{H}_{2} \mathrm{O}$ and $57.05 \mathrm{~g}(0.284 \mathrm{~mol})$ acesulfame-K in $\mathrm{H}_{2}$ O. - Yield $=26.2 \mathrm{~g}(92 \%) .-n_{\mathrm{D}}^{20}=1.5019$. - IR (film): $v=1534\left(\mathrm{CN}_{3}{ }^{+}\right), 1172\left(\mathrm{SO}_{2}\right), 1602(\mathrm{C}=\mathrm{C}), 1650$ $(\mathrm{C}=\mathrm{O}) \mathrm{cm}^{-1} .-{ }^{1} \mathrm{H} \mathrm{NMR}\left(250.13 \mathrm{MHz}, \mathrm{CDCl}_{3}\right): \delta=0.86-$ $1.01\left(\mathrm{~m}, 12 \mathrm{H}, 2 \times \mathrm{C}_{2} \mathrm{H}_{4} \mathrm{CH}_{3}, \mathrm{C}_{3} \mathrm{H}_{6} \mathrm{CH}_{3}, \mathrm{C}_{5} \mathrm{H}_{10} \mathrm{CH}_{3}\right), 1.12-$ $1.90\left(\mathrm{~m}, 22 \mathrm{H}, 8 \times \mathrm{CH}_{2}, 2 \times \mathrm{CH}_{2} \mathrm{CH}_{3}\right), 1.99(\mathrm{~d}, \mathrm{~J}=1.0 \mathrm{~Hz}$, $3 \mathrm{H}, \mathrm{CH}_{3} /$ Ace $), 2.95-3.51\left(\mathrm{~m}, 12 \mathrm{H}\right.$, all $\left.\mathrm{CH}_{2} \mathrm{~N}\right), 5.46(\mathrm{~d}$, $J=1.0 \mathrm{~Hz}, 1 \mathrm{H}, \mathrm{CH} /$ Ace $).-{ }^{13} \mathrm{C} \mathrm{NMR}\left(62.9 \mathrm{MHz}, \mathrm{CDCl}_{3}\right)$ : $\delta=11.3\left(\mathrm{CH}_{3}\right), 11.4\left(\mathrm{CH}_{3}\right), 12.9\left(\mathrm{CH}_{3}\right), 13.7\left(\mathrm{CH}_{3}\right), 13.9$ $\left(2 \times \mathrm{CH}_{3}\right), 20.1\left(\mathrm{CH}_{3} /\right.$ Ace $), 20.9\left(\mathrm{CH}_{2}\right), 21.0\left(\mathrm{CH}_{2}\right), 22.5$ $\left(2 \times \mathrm{CH}_{2}\right), 26.6\left(\mathrm{CH}_{2}\right), 27.4\left(\mathrm{CH}_{2}\right), 29.5\left(\mathrm{CH}_{2}\right), 31.3\left(\mathrm{CH}_{2}\right)$, $44.0\left(2 \times \mathrm{CH}_{2} \mathrm{~N}\right), 49.4\left(\mathrm{CH}_{2} \mathrm{~N}\right), 49.7\left(\mathrm{CH}_{2} \mathrm{~N}\right), 51.4(2 \times$ $\left.\mathrm{CH}_{2} \mathrm{~N}\right), 102.3(\mathrm{CH} /$ Ace $), 160.3(\mathrm{C}-\mathrm{O} /$ Ace $), 163.9\left(\mathrm{CN}_{3}{ }^{+}\right)$, $169.7(\mathrm{C}=\mathrm{O} /$ Ace $)$. - Anal. for $\mathrm{C}_{25} \mathrm{H}_{50} \mathrm{~N}_{4} \mathrm{O}_{4} \mathrm{~S}(502.8) \times 0.5$ $\mathrm{H}_{2} \mathrm{O}$ : calcd. C 58.67, H 10.04, N 10.95; found C 58.35, H 9.88, N 11.19.

\section{$N, N$-Diethyl- $N^{\prime}, N^{\prime}$-di-n-propyl- $N^{\prime \prime}, N^{\prime \prime}$-di-n-}

hexylguanidinium acesulfamate $\left(\boldsymbol{C}_{\mathbf{6}}-\mathbf{g} \boldsymbol{A} \boldsymbol{e}\right)$

This compound was obtained from $22.84 \mathrm{~g}(0.0565 \mathrm{~mol})$ of $\mathbf{C}_{6} \mathbf{- g C l}$ in $\mathrm{H}_{2} \mathrm{O}$ and $22.74 \mathrm{~g}(0.113 \mathrm{~mol})$ acesulfame-K in $\mathrm{H}_{2}$ O. - Yield $=26.5 \mathrm{~g}(88 \%) .-n_{\mathrm{D}}^{20}=1.4994$. - IR (film): $v=1532\left(\mathrm{CN}_{3}{ }^{+}\right), 1172\left(\mathrm{SO}_{2}\right), 1603(\mathrm{C}=\mathrm{C}), 1650$ $(\mathrm{C}=\mathrm{O}) \mathrm{cm}^{-1} .-{ }^{1} \mathrm{H} \mathrm{NMR}\left(250.13 \mathrm{MHz}, \mathrm{CDCl}_{3}\right): \delta=0.86-$ $0.97\left(\mathrm{~m}, 12 \mathrm{H}, 2 \times \mathrm{C}_{2} \mathrm{H}_{4} \mathrm{CH}_{3}, 2 \times \mathrm{C}_{5} \mathrm{H}_{10} \mathrm{CH}_{3}\right), 1.13-1.89$ $\left(\mathrm{m}, 26 \mathrm{H}, 10 \times \mathrm{CH}_{2}, 2 \times \mathrm{CH}_{2} \mathrm{CH}_{3}\right), 1.99(\mathrm{~d}, \mathrm{~J}=1.0 \mathrm{~Hz}$, $3 \mathrm{H}, \mathrm{CH}_{3} /$ Ace $), 2.94-3.51\left(\mathrm{~m}, 12 \mathrm{H}\right.$, all $\left.\mathrm{CH}_{2} \mathrm{~N}\right), 5.48(\mathrm{~d}$, $J=1.0 \mathrm{~Hz}, 1 \mathrm{H}, \mathrm{CH} / \mathrm{Ace}) .-{ }^{13} \mathrm{C} \mathrm{NMR}\left(62.9 \mathrm{MHz}, \mathrm{CDCl}_{3}\right)$ : $\delta=11.3\left(\mathrm{CH}_{3}\right), 11.4\left(\mathrm{CH}_{3}\right), 12.9\left(2 \times \mathrm{CH}_{3}\right), 13.9\left(2 \times \mathrm{CH}_{3}\right)$, $20.0\left(\mathrm{CH}_{3} /\right.$ Ace $), 21.0\left(2 \times \mathrm{CH}_{2}\right), 22.5\left(2 \times \mathrm{CH}_{2}\right), 26.5(2 \times$ $\left.\mathrm{CH}_{2}\right), 27.4\left(2 \times \mathrm{CH}_{2}\right), 31.3\left(2 \times \mathrm{CH}_{2}\right), 44.1\left(2 \times \mathrm{CH}_{2} \mathrm{~N}\right)$, $49.7\left(2 \times \mathrm{CH}_{2} \mathrm{~N}\right), 51.4\left(2 \times \mathrm{CH}_{2} \mathrm{~N}\right), 102.3(\mathrm{CH} /$ Ace $), 160.3$ (C-O/Ace), $163.9\left(\mathrm{~s}, \mathrm{CN}_{3}{ }^{+}\right), 169.7(\mathrm{C}=\mathrm{O} /$ Ace $)$. - Anal. for $\mathrm{C}_{27} \mathrm{H}_{54} \mathrm{~N}_{4} \mathrm{O}_{4} \mathrm{~S}(530.8) \times \mathrm{H}_{2} \mathrm{O}$ : calcd. C 59.09, H 10.28, N 10.21; found C 59.47, H 10.16, N 10.25.

$N, N$-Diethyl- $N^{\prime}, N^{\prime}$-di-n-propyl- $N^{\prime \prime}-n$-hexyl- $N^{\prime \prime}-n$ octylguanidinium acesulfamate $\left(\boldsymbol{C}_{\mathbf{8}} \mathbf{- g}\right.$ Ace $)$

This compound was obtained from $12.37 \mathrm{~g}(0.028 \mathrm{~mol})$ of $\mathbf{C}_{\mathbf{8}} \mathbf{- g C l}$ in $\mathrm{H}_{2} \mathrm{O}$ and $28.8 \mathrm{~g}(0.143 \mathrm{~mol})$ acesulfame-K in $\mathrm{H}_{2}$ O. - Yield $=15.9 \mathrm{~g}(99 \%) .-n_{\mathrm{D}}^{20}=1.4978$. - IR (film): $v=1533\left(\mathrm{CN}_{3}{ }^{+}\right), 1172\left(\mathrm{SO}_{2}\right), 1601(\mathrm{C}=\mathrm{C}), 1650$ $(\mathrm{C}=\mathrm{O}) \mathrm{cm}^{-1} .-{ }^{1} \mathrm{H} \mathrm{NMR}\left(250.13 \mathrm{MHz}, \mathrm{CDCl}_{3}\right): \delta=0.77-$ $1.01\left(\mathrm{~m}, 12 \mathrm{H}, 2 \times \mathrm{C}_{2} \mathrm{H}_{4} \mathrm{CH}_{3}, \mathrm{C}_{5} \mathrm{H}_{10} \mathrm{CH}_{3}, \mathrm{C}_{7} \mathrm{H}_{14} \mathrm{CH}_{3}\right)$, $1.08-1.91\left(\mathrm{~m}, 30 \mathrm{H}, 12 \times \mathrm{CH}_{2}, 2 \times \mathrm{CH}_{2} \mathrm{CH}_{3}\right), 1.99(\mathrm{~d}$, $J=1.0 \mathrm{~Hz}, 3 \mathrm{H}, \mathrm{CH}_{3} /$ Ace $), 3.00-3.52\left(\mathrm{~m}, 12 \mathrm{H}\right.$, all $\left.\mathrm{CH}_{2} \mathrm{~N}\right)$, $5.46(\mathrm{~d}, J=1.0 \mathrm{~Hz}, 1 \mathrm{H}, \mathrm{CH} /$ Ace $) .-{ }^{13} \mathrm{C} \mathrm{NMR}(62.9 \mathrm{MHz}$, $\left.\mathrm{CDCl}_{3}\right): \delta=11.4\left(2 \times \mathrm{CH}_{3}\right), 12.9\left(\mathrm{CH}_{3}\right), 13.0\left(\mathrm{CH}_{3}\right), 13.9$ $\left(\mathrm{CH}_{3}\right), 14.1\left(\mathrm{CH}_{3}\right), 19.9\left(\mathrm{CH}_{3} /\right.$ Ace $), 20.9\left(\mathrm{CH}_{2}\right), 21.0\left(\mathrm{CH}_{2}\right)$, $22.5\left(\mathrm{CH}_{2}\right), 22.6\left(\mathrm{CH}_{2}\right), 26.6\left(\mathrm{CH}_{2}\right), 26.9\left(\mathrm{CH}_{2}\right), 27.5(2 \times$ 
$\left.\mathrm{CH}_{2}\right), 29.2\left(2 \times \mathrm{CH}_{2}\right), 31.3\left(\mathrm{CH}_{2}\right), 31.7\left(\mathrm{CH}_{2}\right), 44.0\left(\mathrm{CH}_{2} \mathrm{~N}\right)$, $44.1\left(\mathrm{CH}_{2} \mathrm{~N}\right), 49.7\left(2 \times \mathrm{CH}_{2} \mathrm{~N}\right), 51.4\left(\mathrm{CH}_{2} \mathrm{~N}\right), 51.5\left(\mathrm{CH}_{2} \mathrm{~N}\right)$, 102.3 (CH/Ace), 160.4 (C-O/Ace), $163.9\left(\mathrm{CN}_{3}{ }^{+}\right), 169.8$ $(\mathrm{C}=\mathrm{O} /$ Ace $)$. - Anal. for $\mathrm{C}_{29} \mathrm{H}_{58} \mathrm{~N}_{4} \mathrm{O}_{4} \mathrm{~S}(558.9) \times \mathrm{H}_{2} \mathrm{O}$ : calcd. C 60.38, H 10.48, N 9.71; found C 60.38, H 10.27, N 9.89 .

$N, N$-Diethyl- $N^{\prime}, N^{\prime}$-di-n-propyl- $N^{\prime \prime}-n$-hexyl- $N^{\prime \prime}-n$ decylguanidinium acesulfamate $\left(\boldsymbol{C}_{10^{-}} \mathbf{g A c e}\right)$

This compound was obtained from $15.62 \mathrm{~g}(0.034 \mathrm{~mol})$ of $\mathbf{C}_{10}$-gCl in $\mathrm{H}_{2} \mathrm{O}$ and $34.21 \mathrm{~g}(0.17 \mathrm{~mol})$ of acesulfame$\mathrm{K}$ in $\mathrm{H}_{2} \mathrm{O}$. - Yield $=19.4 \mathrm{~g}(97 \%) .-n_{\mathrm{D}}^{20}=1.4952$. $-\mathrm{IR}$ (film): $v=1533\left(\mathrm{CN}_{3}{ }^{+}\right), 1172\left(\mathrm{SO}_{2}\right), 1603(\mathrm{C}=\mathrm{C}), 1650$ $(\mathrm{C}=\mathrm{O}) \mathrm{cm}^{-1} .-{ }^{1} \mathrm{H} \mathrm{NMR}\left(250.13 \mathrm{MHz}, \mathrm{CDCl}_{3}\right): \delta=0.79-$ $1.01\left(\mathrm{~m}, 12 \mathrm{H}, 2 \times \mathrm{C}_{2} \mathrm{H}_{4} \mathrm{CH}_{3}, \mathrm{C}_{5} \mathrm{H}_{10} \mathrm{CH}_{3}, \mathrm{C}_{9} \mathrm{H}_{18} \mathrm{CH}_{3}\right)$, $1.12-1.89\left(\mathrm{~m}, 34 \mathrm{H}, 14 \times \mathrm{CH}_{2}, 2 \times \mathrm{CH}_{2} \mathrm{CH}_{3}\right), 2.00$ (d, $J=1.0 \mathrm{~Hz}, 3 \mathrm{H}, \mathrm{CH}_{3} /$ Ace $), 2.88-3.53(\mathrm{~m}, 12 \mathrm{H}$, all $\left.\mathrm{CH}_{2} \mathrm{~N}\right), 5.46(\mathrm{~d}, J=1.0 \mathrm{~Hz}, 1 \mathrm{H}, \mathrm{CH} /$ Ace $) .-{ }^{13} \mathrm{C}$ NMR $\left(62.9 \mathrm{MHz}, \mathrm{CDCl}_{3}\right): \delta=11.3\left(\mathrm{CH}_{3}\right), 11.4\left(\mathrm{CH}_{3}\right), 12.9$ $\left(2 \times \mathrm{CH}_{3}\right), 13.9\left(\mathrm{CH}_{3}\right), 14.1\left(\mathrm{CH}_{3}\right), 19.9\left(\mathrm{CH}_{3} /\right.$ Ace $), 20.9$ $\left(\mathrm{CH}_{2}\right), 22.6\left(\mathrm{CH}_{2}\right), 26.5\left(\mathrm{CH}_{2}\right), 26.6\left(\mathrm{CH}_{2}\right), 26.9\left(2 \times \mathrm{CH}_{2}\right)$, $27.4\left(\mathrm{CH}_{2}\right), 27.5\left(\mathrm{CH}_{2}\right), 29.2\left(\mathrm{CH}_{2}\right), 29.3\left(\mathrm{CH}_{2}\right), 29.5(2 \times$ $\left.\mathrm{CH}_{2}\right), 31.3\left(\mathrm{CH}_{2}\right), 31.8\left(\mathrm{CH}_{2}\right), 43.9\left(\mathrm{CH}_{2} \mathrm{~N}\right), 44.0\left(\mathrm{CH}_{2} \mathrm{~N}\right)$, $49.7\left(2 \times \mathrm{CH}_{2} \mathrm{~N}\right), 51.4\left(2 \times \mathrm{CH}_{2} \mathrm{~N}\right), 102.4(\mathrm{CH} /$ Ace $), 160.4$ (C-O/Ace), $163.9\left(\mathrm{CN}_{3}{ }^{+}\right), 169.8(\mathrm{C}=\mathrm{O} /$ Ace $)$. - Anal. for $\mathrm{C}_{31} \mathrm{H}_{62} \mathrm{~N}_{4} \mathrm{O}_{4} \mathrm{~S}$ (586.9): calcd. C 61.55, $\mathrm{H}$ 10.66, N 9.26; found $\mathrm{C} 61.60, \mathrm{H} 10.55, \mathrm{~N} 9.24$.

\section{$N, N$-Diethyl- $N^{\prime}, N^{\prime}$-di-n-propyl- $N^{\prime \prime}-n$-hexyl- $N^{\prime \prime}-n-$} propylguanidinium saccharinate $\left(\boldsymbol{C}_{\mathbf{3}}-\mathbf{g S a c}\right)$

This compound was obtained from $8.86 \mathrm{~g}(0.025 \mathrm{~mol})$ of $\mathbf{C}_{3}-\mathbf{g C l}$ in $\mathrm{H}_{2} \mathrm{O}$ and $30.6 \mathrm{~g}(0.127 \mathrm{~mol})$ of sodium saccharinate in $\mathrm{H}_{2} \mathrm{O}$. - Yield $=10.3 \mathrm{~g}(82 \%) .-n_{\mathrm{D}}^{20}=1.5304$. $-\mathrm{IR}$ (film): $v=1534\left(\mathrm{CN}_{3}{ }^{+}\right), 1116$ and $1142\left(\mathrm{SO}_{2}\right), 1583(\mathrm{C}=\mathrm{C})$, $1637(\mathrm{C}=\mathrm{O}) \mathrm{cm}^{-1} .-{ }^{1} \mathrm{H}$ NMR $\left(250.13 \mathrm{MHz}, \mathrm{CDCl}_{3}\right): \delta=$ $0.78-0.98\left(\mathrm{~m}, 12 \mathrm{H}, 3 \times \mathrm{C}_{2} \mathrm{H}_{4} \mathrm{CH}_{3}, \mathrm{C}_{5} \mathrm{H}_{10} \mathrm{CH}_{3}\right), 1.09-1.90$ $\left(\mathrm{m}, 2 \mathrm{H}, 7 \times \mathrm{CH}_{2}, 2 \times \mathrm{CH}_{2} \mathrm{CH}_{3}\right), 2.89-3.55(\mathrm{~m}, 12 \mathrm{H}$, all $\left.\mathrm{CH}_{2} \mathrm{~N}\right), 7.46-7.53(\mathrm{~m}, 2 \mathrm{H}, 2 \times \mathrm{CH} / \mathrm{Sac}), 7.66-7.75(\mathrm{~m}$, $1 \mathrm{H}, \mathrm{CH} / \mathrm{Sac}), 7.76-7.83(\mathrm{~m}, 1 \mathrm{H}, \mathrm{CH} / \mathrm{Sac}) .-{ }^{13} \mathrm{C} \mathrm{NMR}$ $\left(62.9 \mathrm{MHz}, \mathrm{CDCl}_{3}\right): \delta=11.3\left(3 \times \mathrm{CH}_{3}\right), 12.9\left(\mathrm{CH}_{3}\right)$, $13.9\left(2 \times \mathrm{CH}_{3}\right), 20.9\left(\mathrm{CH}_{2}\right), 22.5\left(3 \times \mathrm{CH}_{2}\right), 26.6\left(\mathrm{CH}_{2}\right)$, $27.4\left(\mathrm{CH}_{2}\right), 31.3\left(\mathrm{CH}_{2}\right), 44.0\left(2 \times \mathrm{CH}_{2} \mathrm{~N}\right), 49.6\left(\mathrm{CH}_{2} \mathrm{~N}\right)$, $51.3\left(3 \times \mathrm{CH}_{2} \mathrm{~N}\right), 119.4(\mathrm{CH} / \mathrm{Sac}), 123.1(\mathrm{CH} / \mathrm{Sac}), 130.6$ $(\mathrm{CH} / \mathrm{Sac}), 131.3(\mathrm{CH} / \mathrm{Sac}), 135.4(\mathrm{C} / \mathrm{Sac}), 145.3(\mathrm{C}-\mathrm{S} / \mathrm{Sac})$, $163.9\left(\mathrm{CN}_{3}{ }^{+}\right), 170.0(\mathrm{C}=\mathrm{O} / \mathrm{Sac}) .-$ Anal. for $\mathrm{C}_{27} \mathrm{H}_{48} \mathrm{~N}_{4} \mathrm{O}_{3} \mathrm{~S}$ $(508.8) \times 0.5 \mathrm{H}_{2} \mathrm{O}$ : calcd. C 62.88, H 9.54, N 10.82; found C 62.43, H 9.38, N 11.03.

$N, N$-Diethyl- $N^{\prime}, N^{\prime}$-di-n-propyl- $N^{\prime \prime}-n$-hexyl- $N^{\prime \prime}-n$ butylguanidinium saccharinate $\left(\boldsymbol{C}_{\mathbf{4}}-\mathbf{g} \mathrm{Sac}\right)$

This compound was obtained from $14.2 \mathrm{~g}(0.039 \mathrm{~mol})$ of $\mathbf{C}_{4}-\mathbf{g C l}$ in $\mathrm{H}_{2} \mathrm{O}$ and $47.3 \mathrm{~g}(0.196 \mathrm{~mol})$ of sodium saccharinate in $\mathrm{H}_{2} \mathrm{O}$. - Yield $=19.0 \mathrm{~g}(95 \%) .-n_{\mathrm{D}}^{20}=$
1.5274. - IR (film): $v=1533\left(\mathrm{CN}_{3}^{+}\right), 1116$ and $1142\left(\mathrm{SO}_{2}\right)$, $1583(\mathrm{C}=\mathrm{C}), 1637(\mathrm{C}=\mathrm{O}) \mathrm{cm}^{-1} .{ }^{1}{ }^{1} \mathrm{H}$ NMR $(250.13 \mathrm{MHz}$, $\left.\mathrm{CDCl}_{3}\right): \delta=0.77-0.99\left(\mathrm{~m}, 12 \mathrm{H}, 2 \times \mathrm{C}_{2} \mathrm{H}_{4} \mathrm{CH}_{3}, \mathrm{C}_{3} \mathrm{H}_{6} \mathrm{CH}_{3}\right.$, $\left.\mathrm{C}_{5} \mathrm{H}_{10} \mathrm{CH}_{3}\right), 1.09-1.91\left(\mathrm{~m}, 22 \mathrm{H}, 8 \times \mathrm{CH}_{2}, 2 \times \mathrm{CH}_{2} \mathrm{CH}_{3}\right)$, $2.93-3.55\left(\mathrm{~m}, 12 \mathrm{H}\right.$, all $\left.\mathrm{CH}_{2} \mathrm{~N}\right), 7.45-7.54(\mathrm{~m}, 2 \mathrm{H}, 2 \times$ $\mathrm{CH} / \mathrm{Sac}), 7.69-7.76(\mathrm{~m}, 1 \mathrm{H}, \mathrm{CH} / \mathrm{Sac}), 7.77-7.83(\mathrm{~m}$, $1 \mathrm{H}, \mathrm{CH} / \mathrm{Sac}) .-{ }^{13} \mathrm{C}$ NMR $\left(62.9 \mathrm{MHz}, \mathrm{CDCl}_{3}\right): \delta=11.3$ $\left(\mathrm{CH}_{3}\right), 11.4\left(\mathrm{CH}_{3}\right), 12.9\left(\mathrm{CH}_{3}\right), 13.7\left(\mathrm{CH}_{3}\right), 13.9\left(\mathrm{CH}_{3}\right)$, $14.0\left(\mathrm{CH}_{3}\right), 20.1\left(\mathrm{CH}_{2}\right), 20.9\left(\mathrm{CH}_{2}\right), 22.5\left(2 \times \mathrm{CH}_{2}\right)$, $26.6\left(\mathrm{CH}_{2}\right), 27.4\left(\mathrm{CH}_{2}\right), 29.4\left(\mathrm{CH}_{2}\right), 31.3\left(\mathrm{CH}_{2}\right), 44.0$ $\left(\mathrm{CH}_{2} \mathrm{~N}\right), 44.1\left(\mathrm{CH}_{2} \mathrm{~N}\right), 49.7\left(\mathrm{CH}_{2} \mathrm{~N}\right), 50.9\left(\mathrm{CH}_{2} \mathrm{~N}\right), 51.3$ $\left(\mathrm{CH}_{2} \mathrm{~N}\right), 52.4\left(\mathrm{CH}_{2} \mathrm{~N}\right), 119.5(\mathrm{CH} / \mathrm{Sac}), 123.1(\mathrm{CH} / \mathrm{Sac})$, 130.7 (CH/Sac), $131.6(\mathrm{CH} / \mathrm{Sac}), 135.3(\mathrm{C} / \mathrm{Sac}), 145.2$ (C-S/Sac), $164.0\left(\mathrm{CN}_{3}{ }^{+}\right), 169.9(\mathrm{C}=\mathrm{O} / \mathrm{Sac})$. - Anal. for $\mathrm{C}_{28} \mathrm{H}_{50} \mathrm{~N}_{4} \mathrm{O}_{3} \mathrm{~S}(522.8) \times \mathrm{H}_{2} \mathrm{O}$ : calcd. C 62.19, H 9.69, N 10.36; found C 62.48, H 9.42, N 10.88 .

$N, N$-Diethyl- $N^{\prime}, N^{\prime}$-di-n-propyl- $N^{\prime \prime}, N^{\prime \prime}-$ di-nhexylguanidinium saccharinate $\left(\boldsymbol{C}_{\mathbf{6}}-\mathbf{g S a c}\right)$

This compound was obtained from $7.35 \mathrm{~g}(0.018 \mathrm{~mol})$ of $\mathbf{C}_{6}-\mathbf{g C l}$ in $\mathrm{H}_{2} \mathrm{O}$ and $22.0 \mathrm{~g}(0.091 \mathrm{~mol})$ of sodium saccharinate in $\mathrm{H}_{2} \mathrm{O}$. - Yield $=9.4 \mathrm{~g}(94 \%) .-n_{\mathrm{D}}^{20}=1.5211$. $-\mathrm{IR}$ (film): $v=1532\left(\mathrm{CN}_{3}{ }^{+}\right), 1116$ and $1143\left(\mathrm{SO}_{2}\right), 1583(\mathrm{C}=\mathrm{C})$, $1637(\mathrm{C}=\mathrm{O}) \mathrm{cm}^{-1} .-{ }^{1} \mathrm{H}$ NMR $\left(250.13 \mathrm{MHz}, \mathrm{CDCl}_{3}\right): \delta=$ $0.79-1.00\left(\mathrm{~m}, 12 \mathrm{H}, 2 \times \mathrm{C}_{2} \mathrm{H}_{4} \mathrm{CH}_{3}, 2 \times \mathrm{C}_{5} \mathrm{H}_{10} \mathrm{CH}_{3}\right), 1.14-$ $1.88\left(\mathrm{~m}, 26 \mathrm{H}, 10 \times \mathrm{CH}_{2}, 2 \times \mathrm{CH}_{2} \mathrm{CH}_{3}\right), 2.93-3.54(\mathrm{~m}$, $12 \mathrm{H}$, all $\left.\mathrm{CH}_{2} \mathrm{~N}\right), 7.44-7.51(\mathrm{~m}, 2 \mathrm{H}, 2 \times \mathrm{CH} / \mathrm{Sac}), 7.68-$ $7.74(\mathrm{~m}, 1 \mathrm{H}, \mathrm{CH} / \mathrm{Sac}), 7.77-7.83(\mathrm{~m}, 1 \mathrm{H}, \mathrm{CH} / \mathrm{Sac})$. ${ }^{13} \mathrm{C}$ NMR $\left(62.9 \mathrm{MHz}, \mathrm{CDCl}_{3}\right): \delta=11.3\left(\mathrm{CH}_{3}\right), 11.4$ $\left(\mathrm{CH}_{3}\right), 13.0\left(2 \times \mathrm{CH}_{3}\right), 13.9\left(2 \times \mathrm{CH}_{3}\right), 20.9\left(\mathrm{CH}_{2}\right)$, $21.0\left(\mathrm{CH}_{2}\right), 22.5\left(2 \times \mathrm{CH}_{2}\right), 26.5\left(\mathrm{CH}_{2}\right), 26.6\left(\mathrm{CH}_{2}\right), 27.4$ $\left(\mathrm{CH}_{2}\right), 27.5\left(\mathrm{CH}_{2}\right), 31.3\left(2 \times \mathrm{CH}_{2}\right), 44.0\left(\mathrm{CH}_{2} \mathrm{~N}\right), 44.1$ $\left(\mathrm{CH}_{2} \mathrm{~N}\right), 49.7\left(2 \times \mathrm{CH}_{2} \mathrm{~N}\right), 51.4\left(\mathrm{CH}_{2} \mathrm{~N}\right), 51.5\left(\mathrm{CH}_{2} \mathrm{~N}\right)$, $119.4(\mathrm{CH} / \mathrm{Sac}), 123.1(\mathrm{CH} / \mathrm{Sac}), 130.5(\mathrm{CH} / \mathrm{Sac}), 131.2$ $(\mathrm{CH} / \mathrm{Sac}), 135.6(\mathrm{C} / \mathrm{Sac}), 145.4(\mathrm{C}-\mathrm{S} / \mathrm{Sac}), 164.0\left(\mathrm{CN}_{3}{ }^{+}\right)$, $169.9(\mathrm{C}=\mathrm{O} / \mathrm{Sac})$. - Anal. for $\mathrm{C}_{30} \mathrm{H}_{54} \mathrm{~N}_{4} \mathrm{O}_{3} \mathrm{~S}$ (550.8): calcd. C 65.41, H 9.88, N 10.17; found C 65.22, H 9.85, N 10.13.

$N, N$-Diethyl- $N^{\prime}, N^{\prime}$-di-n-propyl- $N^{\prime \prime}-n$-hexyl- $N^{\prime \prime}-n-$ octylguanidinium saccharinate $\left(\boldsymbol{C}_{\mathbf{8}}-\mathbf{g S a c}\right)$

This compound was obtained from $14.9 \mathrm{~g}(0.034 \mathrm{~mol})$ of $\mathrm{C}_{8}$-gCl in $\mathrm{H}_{2} \mathrm{O}$ and $41.7 \mathrm{~g}(0.173 \mathrm{~mol})$ of sodium saccharinate in $\mathrm{H}_{2} \mathrm{O}$. - Yield $=19.8 \mathrm{~g}(99 \%) .-n_{\mathrm{D}}^{20}=1.5202$. $-\mathrm{IR}$ (film): $v=1533\left(\mathrm{CN}_{3}{ }^{+}\right), 1116$ and $1143\left(\mathrm{SO}_{2}\right), 1583(\mathrm{C}=\mathrm{C})$, $1637(\mathrm{C}=\mathrm{O}) \mathrm{cm}^{-1} .-{ }^{1} \mathrm{H}$ NMR $\left(250.13 \mathrm{MHz}, \mathrm{CDCl}_{3}\right)$ : $\delta=0.77-1.00\left(\mathrm{~m}, 12 \mathrm{H}, 2 \times \mathrm{C}_{2} \mathrm{H}_{4} \mathrm{CH}_{3}, \mathrm{C}_{5} \mathrm{H}_{10} \mathrm{CH}_{3}\right.$, $\left.\mathrm{C}_{7} \mathrm{H}_{14} \mathrm{CH}_{3}\right), 1.09-1.91\left(\mathrm{~m}, 30 \mathrm{H}, 12 \times \mathrm{CH}_{2}, 2 \times \mathrm{CH}_{2} \mathrm{CH}_{3}\right)$, $2.88-3.57\left(\mathrm{~m}, 12 \mathrm{H}\right.$, all $\left.\mathrm{CH}_{2} \mathrm{~N}\right), 7.46-7.53(\mathrm{~m}, 2 \mathrm{H}, 2 \times$ $\mathrm{CH} / \mathrm{Sac}$ ), $7.68-7.75$ (m, $1 \mathrm{H}, \mathrm{CH} / \mathrm{Sac}), 7.76-7.84$ (m, $1 \mathrm{H}$, $\mathrm{CH} / \mathrm{Sac}) .-{ }^{13} \mathrm{C}$ NMR $\left(62.9 \mathrm{MHz}, \mathrm{CDCl}_{3}\right): \delta=11.3(2 \times$ $\left.\mathrm{CH}_{3}\right), 12.9\left(2 \times \mathrm{CH}_{3}\right), 13.9\left(\mathrm{CH}_{3}\right), 14.1\left(\mathrm{CH}_{3}\right), 20.9\left(\mathrm{CH}_{2}\right)$, $21.0\left(\mathrm{CH}_{2}\right), 22.6\left(2 \times \mathrm{CH}_{2}\right), 26.6\left(\mathrm{CH}_{2}\right), 26.8\left(\mathrm{CH}_{2}\right), 27.4$ 
$\left(2 \times \mathrm{CH}_{2}\right), 29.1\left(2 \times \mathrm{CH}_{2}\right), 31.2\left(\mathrm{CH}_{2}\right), 31.7\left(\mathrm{CH}_{2}\right), 44.0$ $\left(2 \times \mathrm{CH}_{2} \mathrm{~N}\right), 49.7\left(2 \times \mathrm{CH}_{2} \mathrm{~N}\right), 51.3\left(\mathrm{CH}_{2} \mathrm{~N}\right), 51.4\left(\mathrm{CH}_{2} \mathrm{~N}\right)$, $119.4(\mathrm{CH} / \mathrm{Sac}), 123.1(\mathrm{CH} / \mathrm{Sac}), 130.6(\mathrm{CH} / \mathrm{Sac}), 131.3$ $(\mathrm{CH} / \mathrm{Sac}), 135.3(\mathrm{C} / \mathrm{Sac}), 145.3(\mathrm{C}-\mathrm{S} / \mathrm{Sac}), 163.9\left(\mathrm{CN}_{3}{ }^{+}\right)$, $169.8(\mathrm{C}=\mathrm{O} / \mathrm{Sac}) .-$ Anal. for $\mathrm{C}_{32} \mathrm{H}_{58} \mathrm{~N}_{4} \mathrm{O}_{3} \mathrm{~S}(578.9) \times 1.5$ $\mathrm{H}_{2} \mathrm{O}$ : calcd. C 63.43, H 10.15, N 9.25; found C 63.20, H 9.83, N 9.30 .

\section{$N, N$-Diethyl- $N^{\prime}, N^{\prime}$-di-n-propyl- $N^{\prime \prime}-n$-hexyl- $N^{\prime \prime}-n$ - decylguanidinium saccharinate $\left(\boldsymbol{C}_{\mathbf{1 0}} \mathbf{- g S a c}\right)$}

This compound was obtained from $7.58 \mathrm{~g}(0.016 \mathrm{~mol})$ of $\mathbf{C}_{\mathbf{1 0}}$-gCl in $\mathrm{H}_{2} \mathrm{O}$ and $19.9 \mathrm{~g}(0.082 \mathrm{~mol})$ of sodium saccharinate in $\mathrm{H}_{2} \mathrm{O} .-$ Yield $=9.9 \mathrm{~g}(99 \%) .-n_{\mathrm{D}}^{20}=1.5184$. - IR (film): $v=1533\left(\mathrm{CN}_{3}{ }^{+}\right), 1116$ and $1143\left(\mathrm{SO}_{2}\right), 1583(\mathrm{C}=\mathrm{C})$, $1637(\mathrm{C}=\mathrm{O}) \mathrm{cm}^{-1} .-{ }^{1} \mathrm{H}$ NMR $\left(250.13 \mathrm{MHz}, \mathrm{CDCl}_{3}\right)$ : $\delta=0.78-0.98\left(\mathrm{~m}, 12 \mathrm{H}, 2 \times \mathrm{C}_{2} \mathrm{H}_{4} \mathrm{CH}_{3}, \mathrm{C}_{5} \mathrm{H}_{10} \mathrm{CH}_{3}\right.$, $\left.\mathrm{C}_{9} \mathrm{H}_{18} \mathrm{CH}_{3}\right), 1.11-1.91\left(\mathrm{~m}, 34 \mathrm{H}, 14 \times \mathrm{CH}_{2}, 2 \times \mathrm{CH}_{2} \mathrm{CH}_{3}\right)$, 2.91-3.56 (m, $12 \mathrm{H}$, all $\left.\mathrm{CH}_{2} \mathrm{~N}\right), 7.45-7.53(\mathrm{~m}, 2 \mathrm{H}, 2 \times$ $\mathrm{CH} / \mathrm{Sac}), 7.69-7.75(\mathrm{~m}, 1 \mathrm{H}, \mathrm{CH} / \mathrm{Sac}), 7.76-7.83(\mathrm{~m}$,
$1 \mathrm{H}, \mathrm{CH} / \mathrm{Sac}) .-{ }^{13} \mathrm{C}$ NMR $\left(62.9 \mathrm{MHz}, \mathrm{CDCl}_{3}\right): \delta=11.3$ $\left(\mathrm{CH}_{3}\right), 11.4\left(\mathrm{CH}_{3}\right), 13.0\left(\mathrm{CH}_{3}\right), 13.9\left(\mathrm{CH}_{3}\right), 14.1(2 \times$ $\left.\mathrm{CH}_{3}\right), 20.9\left(\mathrm{CH}_{2}\right), 21.0\left(\mathrm{CH}_{2}\right), 22.5\left(\mathrm{CH}_{2}\right), 22.6\left(\mathrm{CH}_{2}\right), 26.6$ $\left(\mathrm{CH}_{2}\right), 26.9\left(\mathrm{CH}_{2}\right), 27.4\left(2 \times \mathrm{CH}_{2}\right), 29.3\left(2 \times \mathrm{CH}_{2}\right), 29.5$ $\left(2 \times \mathrm{CH}_{2}\right), 31.3\left(\mathrm{CH}_{2}\right), 31.8\left(\mathrm{CH}_{2}\right), 44.0\left(\mathrm{CH}_{2} \mathrm{~N}\right), 44.2$ $\left(\mathrm{CH}_{2} \mathrm{~N}\right), 49.7\left(2 \times \mathrm{CH}_{2} \mathrm{~N}\right), 51.4\left(\mathrm{CH}_{2} \mathrm{~N}\right), 51.5\left(\mathrm{CH}_{2} \mathrm{~N}\right)$, $119.5(\mathrm{CH} / \mathrm{Sac}), 123.1(\mathrm{CH} / \mathrm{Sac}), 130.6(\mathrm{CH} / \mathrm{Sac}), 131.3$ (CH/Sac), $135.4(\mathrm{C} / \mathrm{Sac}), 145.3(\mathrm{C}-\mathrm{S} / \mathrm{Sac}), 163.9\left(\mathrm{CN}_{3}{ }^{+}\right)$, $170.0(\mathrm{C}=\mathrm{O} / \mathrm{Sac}) .-$ Anal. for $\mathrm{C}_{34} \mathrm{H}_{62} \mathrm{~N}_{4} \mathrm{O}_{3} \mathrm{~S}(606.9) \times \mathrm{H}_{2} \mathrm{O}$ : calcd. C 65.34, H 10.32, N 8.96; found C 65.17, H 10.07, N 9.28 .

\section{Acknowledgements}

The present work was funded by the Bundesministerium für Bildung und Forschung (Programm: Innovationen als Schlüssel für Nachhaltigkeit in der Wirtschaft. Verbundprojekt: Neuartige ionische Flüssigkeiten als innovative Reaktionsmedien für die Technische Organische Chemie. Förderkennzeichen 01RI05176). We are indebted to BASF SE, Ludwigshafen, for the gift of chemicals.
[1] a) F. Endres, S. Zein El Abedin, Phys. Chem. Chem. Phys. 2006, 8, 2101-2116; b) P. Wasserscheid, A. Keim, Angew. Chem. 2000, 112, 39263945; Angew. Chem. Int. Ed. 2000, 39, 3772-3789; c) S. A. Forsyth, J. M. Pringle, D. R. MacFarlane, Aust. J. Chem. 2004, 57, $113-119$; d) J. Dupont, P. A.Z. Suarez, Phys. Chem. Chem. Phys. 2006, 8, $2441-$ 2452; e) J. L. Anderson, D. W. Armstrong, Anal. Chem. 2003, 75, $4851-4858$.

[2] P. Wang, S. M. Zakeeruddin, M. Grätzel, W. Kantlehner, J. Mezger, E. V. Stoyanov, O. Scherr, Appl. Phys. A: Mater. Sci. Process. 2004, A79, $73-77$.

[3] Y. Gao, S. W. Arritt, B. Twamley, J. M. Shreeve, Inorg. Chem. 2005, 44, $1704-1712$.

[4] a) N. M. M. Mateus, L.C. Branco, N. M. T. Lourenço, C. A. M. Afonso, Green Chem. 2003, 5, 347-352; b) P. S. Kulkarni, L. C. Branco, J. G. Crespo, M. Cristiana Nunes, A. Raymundo, C. A. M. Afonso, Chem. Eur. J. 2007, 13, 8478-8488; c) L. C. Branco, P. M. P. Gois, N. M. T. Lourenc, V. B. Kurteva and C. A. M. Afonso, Chem. Commun. 2006, 2371-2372.

[5] H. Kunkel, G. Maas, Eur. J. Org. Chem. 2007, $3746-$ 3757.

[6] J. Shah, J. Liebscher, Synthesis 2008, 917-920.

[7] a) S. Fang, L. Yang, C. Wei, C. Jiang, K. Tachibana, K. Kamijima, Electrochim. Acta 2009, 54, $1752-$ 1756; b) S. Fang, L. Yang, J. Wang, H. Zhang, K. Tachibana, K. Kamijima, J. Power Sour. 2009, 191, 619622.

[8] D. Li, M. Wang, J. Wu, Q. Zhang, Y. Luo, Z. Yu, Q. Meng, Z. Wu, Langmuir 2009, 25, 4808-4814.
[9] a) W. Kantlehner, H. Hagen, German Patent (DOS) 2716 477, BASF AG 1978; C. A. 90, 38552 (1979); b) W. Kantlehner, H. Hagen, German Patent (DOS) 2718 275, BASF AG 1978; C. A. 90, 86 777 (1979); c) W. Kantlehner, L. Kienitz, H. Jaus, H. Bredereck, Liebigs Ann. Chem. 1979, 2089-2095; d) W. Kantlehner, E. Haug, W. W. Mergen, P. Speh, T. Maier, J. J. Kapassakalidis, H. J. Bräuner, H. Hagen, Synthesis 1983, 904-905; e) W. Kantlehner, E. Haug, W. W. Mergen, P. Speh, T. Maier, J. J. Kapassakalidis, H. J. Bräuner, H. Hagen, Liebigs Ann. Chem. 1984, $108-126$.

[10] S. V. Dzyuba, R. A. Bartsch, J. Heterocyclic Chem. 2001, 38, 265-268.

[11] C. M. Gordon, M. J. Muldoon in Ionic Liquids in Synthesis, $2^{\text {nd }}$ Ed. (Eds.: P. Wasserscheid, T. Welton), Wiley-VCH, Weinheim, 2008, pp. 10.

[12] A. G. Böwing, A. Jess, Green Chem. 2005, 7, $230-$ 235.

[13] M. B. Smith, J. March, March's advanced organic chemistry: reactions, mechanisms, and structure, $5^{\text {th }}$ ed., John Wiley \& Sons, New York, 2001, pp. 450.

[14] J. G. Huddleston, H. D. Willauer, R. P. Swatloski, A. E. Visser, R. D. Rogers, Chem. Commun. 1998, $1765-$ 1766.

[15] H. Ohno, K. Fukumoto, Acc. Chem. Res. 2007, 40, $1122-1129$.

[16] M. G. Bogdanov, W. Kantlehner, Z. Naturforsch. 2009, $64 b, 215-222$.

[17] M. G. Bogdanov, B. Iliev, W. Kantlehner, Z. Naturforsch. 2009, 64b, 756-764. 